\title{
Turbulent transport in the outer region of rough-wall open-channel flows: the contribution of large coherent shear stress structures (LC3S)
}

\author{
D. HURTHER ${ }^{1}$, U. LEMMIN ${ }^{2}$ AND E. A. TERRA Y \\ ${ }^{1}$ Laboratoire des Ecoulements Géophysiques et Industriels, CNRS UMR 5519, \\ Grenoble, 38041, France \\ ${ }^{2}$ Ecole Polytechnique Fédérale Lausanne, CH-1015 Lausanne, Switzerland \\ ${ }^{3}$ Woods Hole Oceanographic Institution, Woods Hole, MA 02543, USA
}

(Received 7 July 2005 and in revised form 27 September 2006)

Acoustic Doppler velocity profiler (ADVP) measurements of instantaneous threedimensional velocity profiles over the entire turbulent boundary layer height, $\delta$, of rough-bed open-channel flows at moderate Reynolds numbers show the presence of large scale coherent shear stress structures (called LC3S herein) in the zones of uniformly retarded streamwise momentum. LC3S events over streamwise distances of several boundary layer thicknesses dominate the mean shear dynamics. Polymodal histograms of short streamwise velocity samples confirm the subdivision of uniform streamwise momentum into three zones also observed by Adrian et al. (J. Fluid Mech., vol. 422, 2000, p. 1). The mean streamwise dimension of the zones varies between $1 \delta$ and $2.5 \delta$. In the intermediate region $(0.2<z / \delta<0.75)$, the contribution of conditionally sampled $u^{\prime} w^{\prime}$ events to the mean vertical turbulent kinetic energy (TKE) flux as a function of threshold level $H$ is found to be generated by LC3S events above a critical threshold level $H_{\max }$ for which the ascendant net momentum flux between LC3S of ejection and sweep types is maximal. The vertical profile of $H_{\max }$ is nearly constant over the intermediate region, with a value of 5 independent of the flow conditions. Very good agreement is found for all flow conditions including the free-stream shear flows studied in Adrian et al. (2000). If normalized by the squared bed friction velocity, the ascendant net momentum flux containing $90 \%$ of the mean TKE flux is equal to $20 \%$ of the shear stress due to bed friction. In the intermediate region this value is nearly constant for all flow conditions investigated herein. It can be deduced that free-surface turbulence in open-channel flows originates from processes driven by LC3S, associated with the zonal organization of streamwise momentum. The good agreement with mean quadrant distribution results in the literature implies that LC3S identified in this study are common features in the outer region of shear flows.

\section{Introduction}

Over the past forty years, research on wall-bounded shear flows has dealt with the dynamics of intermittent quasi-organized flow events occurring on scales that vary with distance from the wall. It is now recognized that these features, known as streaks, omega vortices, hairpin structures, bursts, vortex heads, ejections, sweeps, boils, upwellings, downwellings, and spiral eddies, are consistent with the presence of clusters, or groups of hairpin vortices, that travel together as a discernible 'packet'. This idea originates from the work of Theodorsen (1952), who proposed a model of the wall layer $\left(z^{+}<100\right.$, where $z^{+}=u_{*} z / v$ is the coordinate normal to the wall 
normalized by the viscous length scale) in which near-wall turbulence is composed of individual symmetric hairpin vortices. The term 'hairpins', which was generalized to include highly elongated asymmetric structures, was subsequently employed by a number of investigators to describe observations obtained from flow visualization. A comprehensive review of work prior to 1990 is given by Robinson (1991).

Most of the studies on coherent structures emphasize flow organization in the inner region of the boundary layer, although visualization experiments show that coherent structures are also found in the outer layer (Brown \& Thomas 1997; Head \& Bandyopadhyay 1981). Adrian, Meinhart \& Tomkins (2000) suggested that these structures were likely to be hairpin packets. This concept was supported by Christensen \& Adrian (2001) who used linear stochastic estimates to construct the velocity field associated with spanwise vortex cores and found patterns consistent with vortex packets. Reynolds numbers in these laboratory experiments were moderate. The major advantage of the hairpin packet model lies in its ability to explain most of the conceptual models of the bursting phenomenon in the inner region as well as the presence of coherent vortical structures in the outer region which scale with the outer parameters of the boundary layer. The change from inner to outer scaling with increasing distance from the wall is thereby taken into account.

Since the concepts presented in Adrian et al. (2000, hereinafter referred to as AMT00), are central to our work, we review them briefly. They propose a subdivision of a boundary layer at any instant into three zones of approximately uniform momentum, which they designate as Zones 1, 2 and 3. This partitioning is based on the observation that a boundary layer consists of relatively large, irregular regions separated by strongly strained layers in which the streamwise flow varies weakly with height above the wall (Meinhart \& Adrian 1995). Coherent groups of hairpin vortices are observed to align themselves in the streamwise direction with their heads at the (typically inclined) boundary between zones. Each hairpin locally induces a retarded flow beneath it. However, the aggregation of vortices within a group creates a long streamwise region of low-momentum fluid whose longitudinal scale is much larger than the vertical or transverse scales of the individual hairpin vortices. The thickness of the three zones (in viscous wall units) depends on the Reynolds number, but the mean streamwise velocity (relative to the free-stream velocity) in the various zones is nearly independent of the Reynolds number.

Zone 1 starts at the beginning of the logarithmic layer, and typically is a few hundred wall units thick. The mean streamwise velocity in this zone is about $60 \%$ of the free-stream speed, $U_{\infty}$. Zone 2 in which the mean speed is about $0.78 U_{\infty}$ starts within the log layer and extends into the wake region. Zone 3 fills the remaining part of the boundary layer. The velocity in this zone is within $5 \%$ of the free-stream velocity. The packets observed in Zone 3 are older than Zone 2 packets and therefore have been generated upstream. Note that packets can be nested, as larger faster moving packets overtake younger packets that are closer to the wall, and hence are moving more slowly. The mean streamwise length of these packets can be several times the boundary layer thickness. The growth angle of a packet is smaller than that of the individual hairpin vortices forming the group. Close to the head, the inclination of individual hairpins varies between $45^{\circ}$ and $90^{\circ}$, and appears to increase with distance from the wall, whereas the inclination of the packet varies between $3^{\circ}$ and $35^{\circ}$, with a mean value of around $10^{\circ}$ which is approximately constant across the entire outer region.

The contribution of hairpin packets to momentum transport in the outer part of the boundary layer has been addressed in several studies (Liu, Adrian \& Hanratty 
2001; Ganapathisubramani, Longmire \& Marusic 2003). Liu et al. applied a proper orthogonal decomposition to velocities in the streamwise-wall-normal plane of a rectangular channel flow at Reynolds numbers of 5378 and 29935 (based on channel half-height). They found that in the outer region, the first 12 eigenmodes carry $48 \%$ to $50 \%$ of the energy and $67 \%$ to $75 \%$ of the Reynolds stress at low and high Reynolds numbers, respectively. Significantly, they found an order of magnitude disparity between the streamwise and wall-normal motions. This difference in scales was interpreted to be a consequence of the hairpin packet organization in which the coherent train of eddies induces an elongated region of reduced $u$-velocity. Ganapathisubramani et al. (2003) used stereo particle image velocimetry (PIV) and a feature extraction algorithm to elucidate the spanwise structure of the packets at several heights within the logarithmic layer. They found that clearly identifiable hairpin packets occupied only $4 \%$ and $4.5 \%$ at heights of $z^{+}=92$ and 150 , respectively of the spanwise plane, but this explained $27 \%$ and $24 \%$ of the Reynolds stress. The relationship between these results and those of Liu et al. is not clear. However, Ganapathisubramani et al. note that the Reynolds stress fraction, which they observed to be associated with hairpin packets, depends on a threshold in their feature detection algorithm. Reducing this by a factor of 4 increases the Reynolds stress contribution by as much as $55 \%$. The question of spanwise structures was addressed by Tomkins \& Adrian (2003). Using PIV, they showed highly elongated streamwise regions of low-momentum fluid at several levels in the outer layer.

The aim of this study is to link recent shear flow organization concepts discussed above with the momentum and turbulence kinetic energy (TKE) budgets in the outer region of open-channel flows. We will divide the boundary layer into an inner region $(z / \delta<0.2)$ and an outer region $(z / \delta>0.2)$. Following Nezu \& Nakagawa (1993) the outer region of open-channel flows is composed of the intermediate region (roughly $0.2<z / \delta<0.75$ ) and the surface-influenced layer (SIL, $z / \delta>0.75$ ). Please note that the region intervals are rough approximations generally used for openchannel flows. They may differ slightly from other definitions found in the literature. We are interested in the hydrodynamic processes at the origin of TKE in the SIL. The link between bed and free-surface turbulence is of particular importance for the hydrodynamic parameterization of turbulence in models of processes occurring at the surface in shallow geophysical flows.

Examples of such processes are the exchange of low-solubility gases, such as carbon dioxide and oxygen across the atmosphere-ocean interface under conditions of strong tidal forcing (Nimmo Smith, Thorpe \& Graham 1999), and the horizontal dispersion of buoyant material (Thorpe et al. 1994; Nimmo Smith et al. 1999). Nimmo Smith et al. (1999) show an example of boils (corresponding to large-scale coherent turbulent flow structures impinging on the free surface) in the North Sea reaching the surface from a $30 \mathrm{~m}$ depth and argue that the dispersion of material on the surface due to currents exceeds that due to wind- and wave-driven Langmuir circulation when the current speed is greater than about $2 \%$ of the wind speed. The effect of wall-generated turbulence on a free surface is also important in river re-oxygenation (Moog \& Jirka 1999), and for the transport of dissolved matter in partially filled large pipes at high discharge levels (Kumar, Gupta \& Banerjee 1998). The mechanism by which momentum and kinetic energy are transported to the SIL is not yet well understood.

In the experimental work to be reported here, we first investigate recent flow organization concepts in the outer region of three turbulent open-channel flows at Reynolds numbers of the order of $10^{4}-10^{5}$ and under three bed roughness conditions, one transitional and two fully rough. The analysis will focus on the existence of 


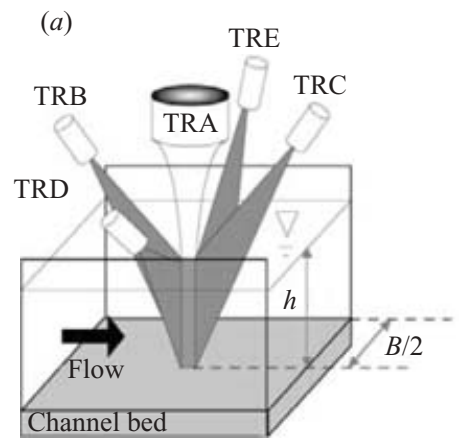

(b)
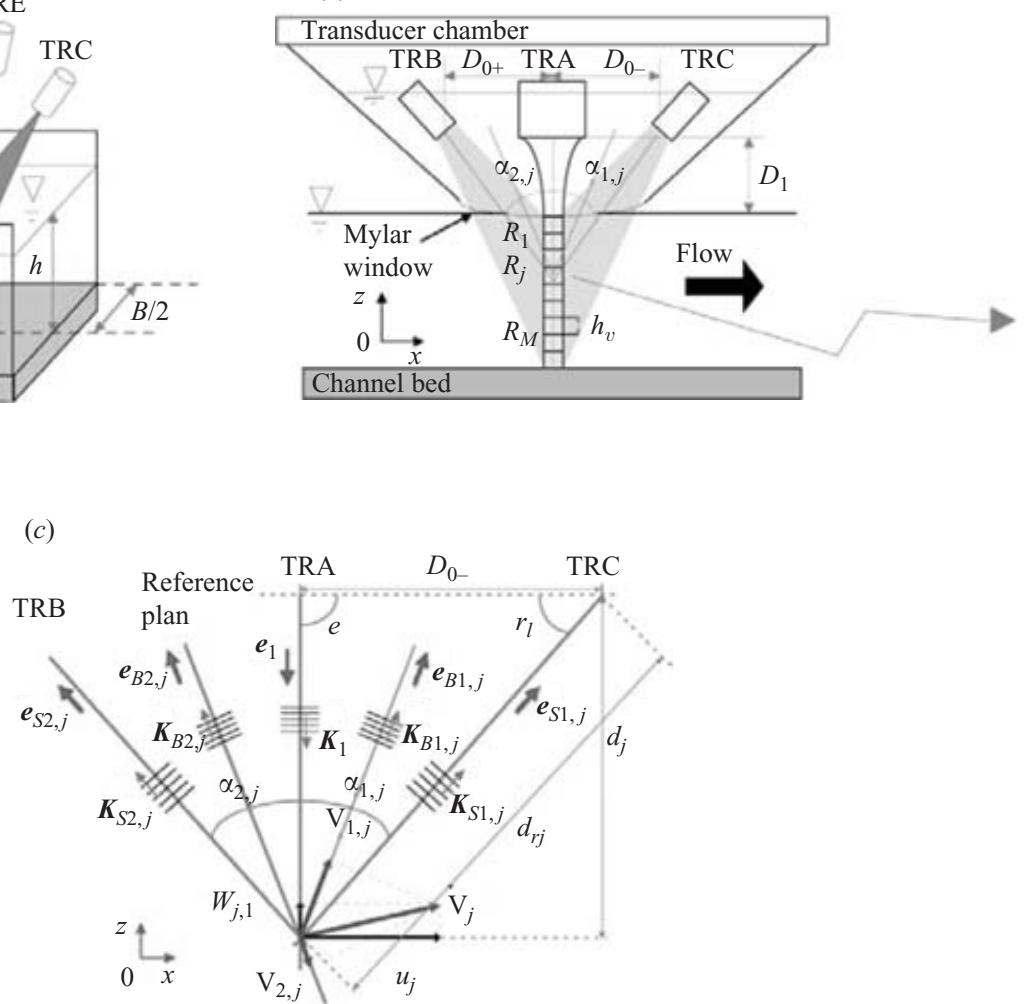

FIgURE 1. (a) Set-up of the high-resolution three-dimensional acoustic Doppler velocity profiler. TRA is a focused phase array emitter. TRB, TRC, TRD, and TRE are the four largeangle receivers arranged to compose two tristatic subsystems in the longitudinal and transverse flow sections. (b) Longitudinal subsystem. (c) Local velocity vector decomposition for the longitudinal tristatic subsystem.

large coherent shear stress structures (LC3S) and their role in the transport of TKE between the wall layer and the SIL. In order to validate the results for the present flow conditions, the same quantitative analysis will also be applied to a low and high Reynolds number flow studied in AMT00. The data for this study were collected with a PIV system in a free-stream shear boundary layer. They are available for downloading at: http://ltcf.tam.uiuc.edu/Downloads/Data/BL/index.html.

\section{Experimental set-up}

\subsection{High-resolution three-dimensional acoustic Doppler velocity profiler (3D ADVP)}

Three-dimensional turbulent velocity profiles were measured along a vertical line over the range $0.02<z / \delta<0.95$ using an acoustic Doppler velocity profiler (ADVP). This type of instrument and its use in measuring turbulent flows are discussed by Garbini, Forster \& Jorgensen (1982), Lhermitte \& Lemmin (1994), Zedel et al. (1996), Hurther \& Lemmin (1998, 2000, 2001, 2003) and Blanckaert \& deVriend (2004, 2005). A schematic of the instrument is shown in figure 1. Four receiving transducers, denoted TRB, TRC, TRD, TRE with a large aperture along the transmit beam receive the backscattered echo from a succession of ranges as the pulse propagates outward. 


\begin{tabular}{lccccccccc}
\hline Run & $\delta(\mathrm{cm})$ & $\theta(\mathrm{cm})$ & $U\left(\mathrm{~cm} \mathrm{~s}^{-1}\right)$ & $u_{*}\left(\mathrm{~cm} \mathrm{~s}^{-1}\right)$ & $R e_{\delta}\left(\times 10^{3}\right)$ & $R e_{\theta}\left(\times 10^{3}\right)$ & $k_{s}^{+}$ & $\delta^{+}$ & $\lambda(\mathrm{cm})$ \\
A & 17.2 & 1.98 & 40 & 2.8 & 68.4 & 7.9 & 280 & 4816 & 1.47 \\
B & 10.6 & 1.2 & 32 & 1.8 & 33.9 & 1.3 & 41 & 1908 & 1.1 \\
C & 16.5 & 1.4 & 49 & 3.1 & 59.4 & 6.8 & 73 & 5110 & 1.4 \\
AH & 7.8 & 0.97 & 1088 & 40 & 55.1 & 6.8 & $<5$ & 2000 & 0.78 \\
AL & 7.57 & 0.91 & 160 & 7.4 & 7.7 & 0.9 & $<5$ & 355 & 1.13
\end{tabular}

TABLE 1. Summary of flow conditions for the present experiments and Adrian et al. (2000).

As shown in figure $1(b)$, the transducers were located in a water-filled chamber, and were separated from the flow by an acoustically transparent Mylar window having a diameter of $15 \mathrm{~cm}$. Hence the measurements were non-invasive, except for the change in boundary conditions under the transducer chamber. For the flow velocities given in table 1, the thickness of the boundary layer at the centre end of the window was estimated to be about $2 \mathrm{~mm}$. The location of the range cell nearest to the window for which data were analysed was 2 to 3 times greater than this thickness. The perturbation due to the transducer chamber was therefore limited to the first sampling volume.

Each emitter-receiver pair formed a bistatic system that measured a profile of the local radial velocity. At each range cell, this was given by the projection of the instantaneous local velocity vector on the axis defined by the Bragg wavenumber vector, which is equal to the difference between the backscattered and the incident vector wavenumbers (see figure $1 c$ ). The local Doppler angle was deduced by triangulation from distance $D_{0}$, angle $e$ and the travel time of the received pulse between the emitter and the receiver. The duration, carrier frequency and pulse repetition frequency of the emitted pulses were equal to $4 \mu \mathrm{s}, 1 \mathrm{MHz}$, and $666 \mathrm{~Hz}$, respectively. This resulted in a spatial resolution of $3 \mathrm{~mm}$ and $7 \mathrm{~mm}$ in the vertical and horizontal directions, respectively, and a temporal resolution of $24 \mathrm{~ms}$ for velocities estimated from 16 consecutive pings. For the flow conditions studied herein these resolutions correspond roughly to 10 wall units in all directions, allowing the determination of the Taylor microscale. The use of the Doppler noise decorrelation technique described in Hurther \& Lemmin (2001) and Blanckaert \& Lemmin (2006), permitted the estimate of the TKE dissipation peak in its spectrum. The Kolmogorov scales could not be resolved in the velocity spectra, but could be calculated from the TKE dissipation rate.

The time-averaged quantities presented in the following sections were all calculated from data samples of $600 \mathrm{~s}$ duration. We estimated the random errors in velocity variances and Reynolds stress for this record length to be in the range of $3 \%$ to $5 \%$ (Soulsby 1980). Hurther \& Lemmin (2001) presented a novel correction method for turbulence measurement with ultrasonic Doppler velocimeters. Following this method, we estimated the residual biases in TKE, stress and TKE flux to be roughly $15 \%, 10 \%$ and $20 \%$, respectively.

\subsection{Flow conditions}

The experiments described here were carried out at the Ecole Polytechnique Fédérale de Lausanne in a rectangular flume $29 \mathrm{~m}$ long, $2.45 \mathrm{~m}$ wide and $0.8 \mathrm{~m}$ high. The channel bed was composed of loose silicium sand representing gravel beds found in rivers. The working section was located $13 \mathrm{~m}$ downstream of the channel's entrance, where the turbulent boundary layer is fully developed. A zero-pressure-gradient condition (or uniform-flow condition) was established over a $2 \mathrm{~m}$ long working section. The origin of the orthogonal coordinate system was located at the bed with the $x$-, $y$ - and 
$z$-axes oriented along the streamwise, transverse and bed-normal directions; $u, v, w$, are the respective instantaneous velocity components.

The three open-channel flows investigated here are denoted as runs A, B and C, and their hydraulic parameters are given in table 1. We have included the AMT00 high and low Reynolds number flow cases in table 1 as runs AH and AL, respectively, in order to compare our flows with their wind-tunnel experiments. The aspect ratio, $W / \delta$, was kept higher than 14 for both runs (here $W$ is the width of the channel and $\delta$ denotes the thickness of the turbulent boundary layer, which we take to be $99 \%$ of the flow depth) in order to avoid perturbations due to secondary circulations induced by anisotropic turbulence interactions between the bed and the sidewalls of the channel (Nezu \& Nakagawa 1993). The flow along the centreline of the channel was therefore two-dimensional in the mean.

As shown in table 1 , the Reynolds numbers of runs $\mathrm{A}, \mathrm{B}$ and $\mathrm{C}$ were moderate and had the same order of magnitude as run AH. The Taylor microscale (determined by the expansion of the autocorrelation function around zero) for runs A, B, C and $\mathrm{AH}$ is similar. From the relative roughness number $k_{s}^{+}=u_{*} k_{s} / v$ (equivalent to a Reynolds roughness number) the beds in runs $\mathrm{A}$ and $\mathrm{C}$ were completely rough $\left(k_{s}^{+}>70\right)$, whereas B was transitionally rough $\left(40<k_{s}^{+}<70\right)$. AMT00's experiments were conducted over smooth beds. Although not shown in table 1, the Shields parameter of all runs was much lower than the critical condition for the initiation of bed motion. Furthermore, no bedforms, ripples, dunes or antidunes were observed at any location in the streamwise direction since the Froude numbers were always less than 0.4 . The parameter $\theta$ in table 1 corresponds to the momentum thickness.

The loose bed material used consisted of silicium sand having a mean grain size of $10 \mathrm{~mm}$ for run $\mathrm{A}$ and $2.3 \mathrm{~mm}$ for runs $\mathrm{B}$ and $\mathrm{C}$. In table $1, \theta$ denotes the momentum thickness, $u_{*}$ is the mean bed friction velocity at the centreline of the channel, and $\delta^{+}$is the boundary layer thickness normalized by the viscous length scale, $v / u_{*}$. The friction velocity was estimated by linearly extrapolating the measured shear stress to the bed. It agrees to within $15 \%$ with the theoretical value, $u_{*}=\sqrt{g S_{0} \delta}$, where $S_{0}$ is the bed slope. This result confirms the uniformity of the flow and indicates the high accuracy of the ADVP in measuring shear stress directly.

The mean downstream Reynolds stress, and the $u^{\prime}-w^{\prime}$ correlation coefficient are shown in figure 2. The measured profiles of mean shear stress for all runs were approximately linear over the region extending from $z / \delta=0.15$ to the free surface. The correlation coefficients were nearly constant over the range $0.2<z / \delta<0.6$. These results are representative of fully developed shear boundary layers similar to the shear flows studied in Raupach (1981), Nezu \& Nakagawa (1977) and AMT00. The differences in the inner region of the shear maxima and their locations are due to significant differences in wall roughness conditions in terms of regime and type.

\section{Large coherent shear stress structures (LC3S) in the outer region}

\subsection{Zones of uniform momentum}

AMT00 showed that the spatial coherence in retarded streamwise velocity (relative to the depth- and time-averaged velocity) over several boundary layer thicknesses results from the streamwise alignment of several hairpin vortices within the envelope of a packet. Three zones of uniform momentum are identified in the outer layer. As seen in figures $3(a)$ and $3(b)$, we observe a similar zonal arrangement in the open-channel flows of runs $\mathrm{A}$ and $\mathrm{B}$, respectively. The grey zones represent regions of uniform streamwise velocity defined as $\left(u-U_{d}\right) / u_{*}$, where $u$ denotes the instantaneous streamwise velocity, and $U_{d}$ is the time-averaged depth-averaged velocity. Three range 


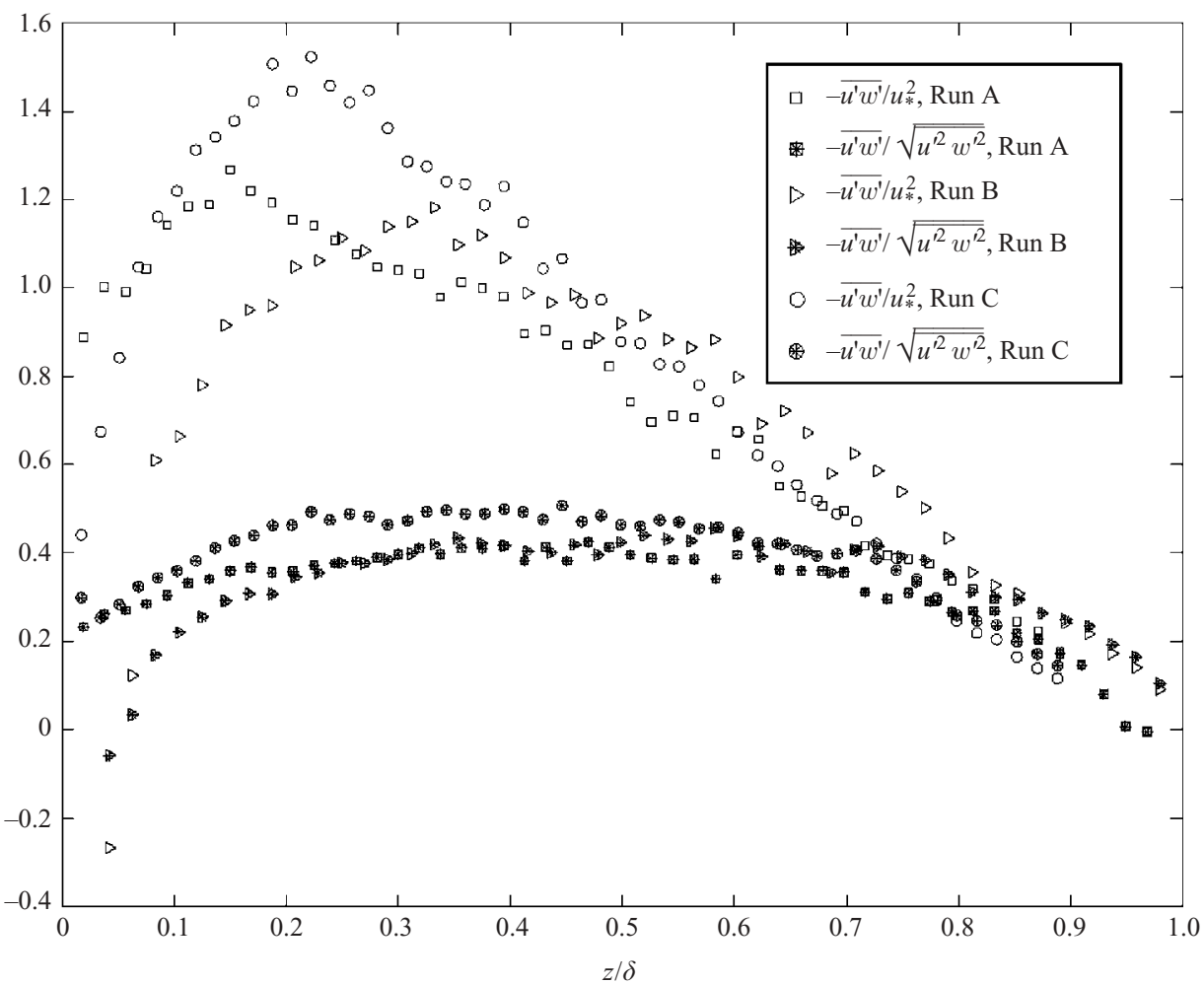

FIGURE 2. Relative shear stress and correlation coefficient profiles for runs A, B and C.
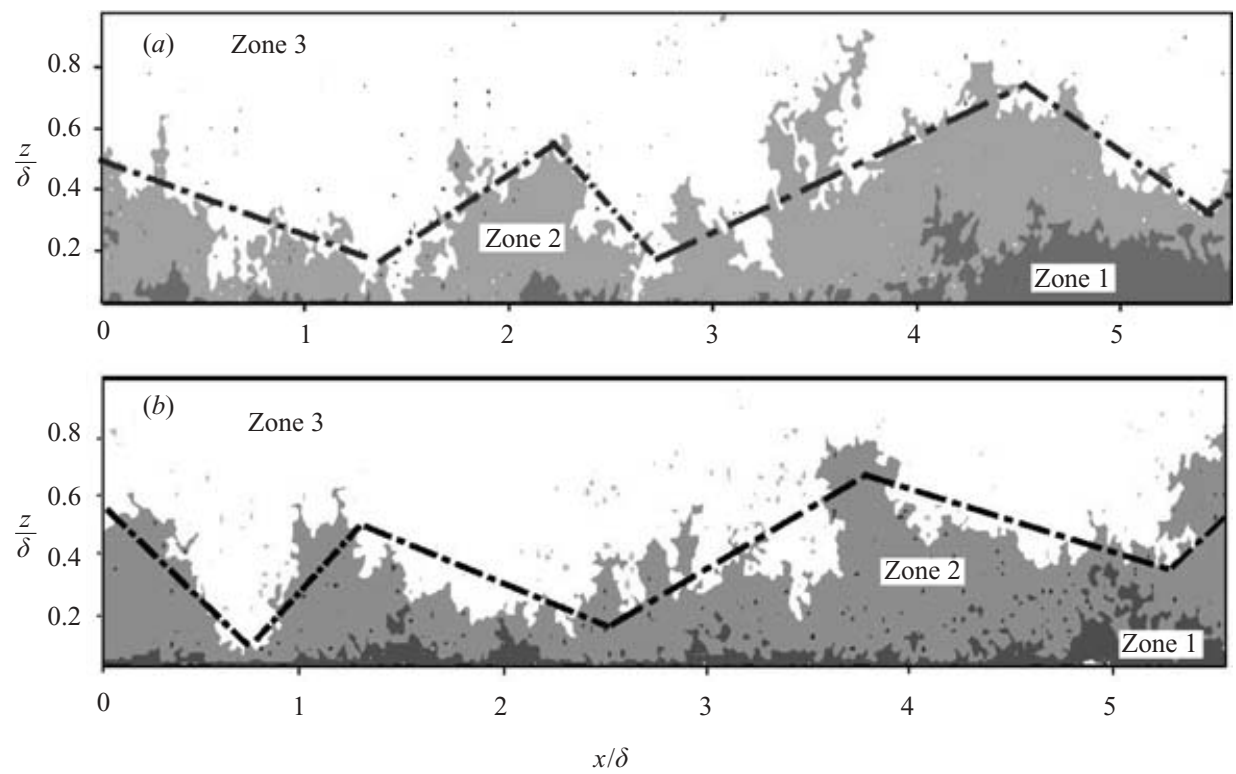

FiguRE 3. Representation of zones of retarded streamwise momentum and fitted separation contours between Zones 2 and 3, $(a)$ for run A, $(b)$ for run B. The grey zones are defined as $\left(u-U_{d}\right) / u_{*}$, where $u$ denotes the instantaneous streamwise velocity, and $U_{d}$ is the time-averaged, depth-averaged velocity. Three range levels are represented. Dark grey corresponds to values of $\left(u-U_{d}\right) / u_{*}$ less than -2.5 , light grey to the range -2.5 to -1.8 , and white indicates values greater than -1.8 . 
levels are represented. Dark grey corresponds to values of $\left(u-U_{d}\right) / u_{*}$ less than -2.5 , light grey to the range -2.5 to -1.8 , and white indicates values greater than -1.8 . For both runs we see regions of uniform streamwise retarded momentum with similar appearance to the zones shown by AMT00. The range of values in the different zones agrees with those for experiment AH of AMT00. It was shown in AMT00 that the length of the packets in Zone 2 can be estimated from the length over which the firstorder curve fitting of the separation contour between Zones 2 and 3 is monotonic. The dash-dotted lines in figures $3(a)$ and $3(b)$ represent those approximations for our experiments. We find values ranging from 1 to $2.5 \delta$ for both runs. Following AMT00's result, the growth angle of these lines is representative for the growth angle of individual packets. In Zone 2, we find angles with a value of $15^{\circ} \pm 6^{\circ}$ (one standard deviation value). Growth angles in Zone 1 are difficult to estimate because the contours between Zone 1 and Zone 2 are not continuous (see figure 3). AMT00 argued that packets in Zone 1 are still in their formation phase and the streamwise alignment of single hairpin vortices is less established than in Zones 2 and 3. As a consequence the inclination of the separation contour between Zones 1 and 2 will mostly depend on the orientation of single hairpin vortices, which varies as a function of depth over a wider range between $0^{\circ}$ and $90^{\circ}$.

The presentation over several boundary layer thicknesses in figure $3(a)$ and $3(b)$ reveals that a positively inclined fitted contour (dash-dotted line) almost always follows a negatively inclined one, giving rise to a 'tent'-like structure with pseudo-cyclic recurrence. The associated wavelength corresponds to the streamwise size of Zone 2.

A more quantitative proof of the existence of zones of uniform streamwise momentum can be seen in the histogram of the instantaneous longitudinal velocity. In order to detect the modal velocities for the different zones in the histogram, the length of the data sample should be of the order of $\delta$. The histogram must be computed from the instantaneous velocity simultaneously measured over the entire boundary layer. The profiling ability of the ADVP can provide the necessary data base.

The resultant histograms for runs $\mathrm{A}$ and $\mathrm{B}$ are shown in figures 4 and 5 , respectively. Three local maxima can be distinguished in both figures. For run A: $U_{z 1} / U_{\infty}=$ $0.45, U_{z 2} / U_{\infty}=0.79, U_{z 3} / U_{\infty}=0.97$, and for run B: $U_{z 1} / U_{\infty}=0.55, U_{z 2} / U_{\infty}=0.83$, $U_{z 3} / U_{\infty}=0.97$, where $U_{\infty}$ is the time-averaged velocity at the free surface of the flow. These velocities correspond to the first-order moments of the three probability density functions (p.d.f.s), denoted by solid lines in the figures. It should be noted that the zonal velocity histograms vary from case to case since the probability of selecting a data sample of length $\delta$ in which all the different zones are well developed is low. AMT00 measured similar polymodal p.d.f.s and corresponding modal velocities $\left(U_{z 1} / U_{\infty}=0.59, U_{z 2} / U_{\infty}=0.76, U_{z 3} / U_{\infty}=0.967\right)$ in the $\mathrm{AH}$ experiment.

The mean locations of the separation contours between the different zones are given in the upper portions of the figures. They were taken to be the normalized depths in the mean longitudinal velocity profiles corresponding to the intersection points between the approximated p.d.f.s. Note that the separation lines between Zones 2 and 3 for runs $\mathrm{A}$ and $\mathrm{B}$ are located at similar depths in the boundary layer $(z / \delta=0.61$ and $z / \delta=0.64$, respectively), whereas the relative difference between the locations of the separation contours between Zones 1 and 2 is equal to $75 \%$ when normalized by the outer-flow scale $\delta(z / \delta=0.05$ and $z / \delta=0.21$, respectively $)$ and reduced to $29 \%$ if normalized by the inner-flow scale $v / u_{*}\left(z^{+}=265\right.$ and $z^{+}=378$ in wall units for runs $\mathrm{A}$ and $\mathrm{B}$, respectively).

In the present section we have shown the presence of zones of uniform streamwise momentum in the outer region of open-channel flows. Their statistical properties 


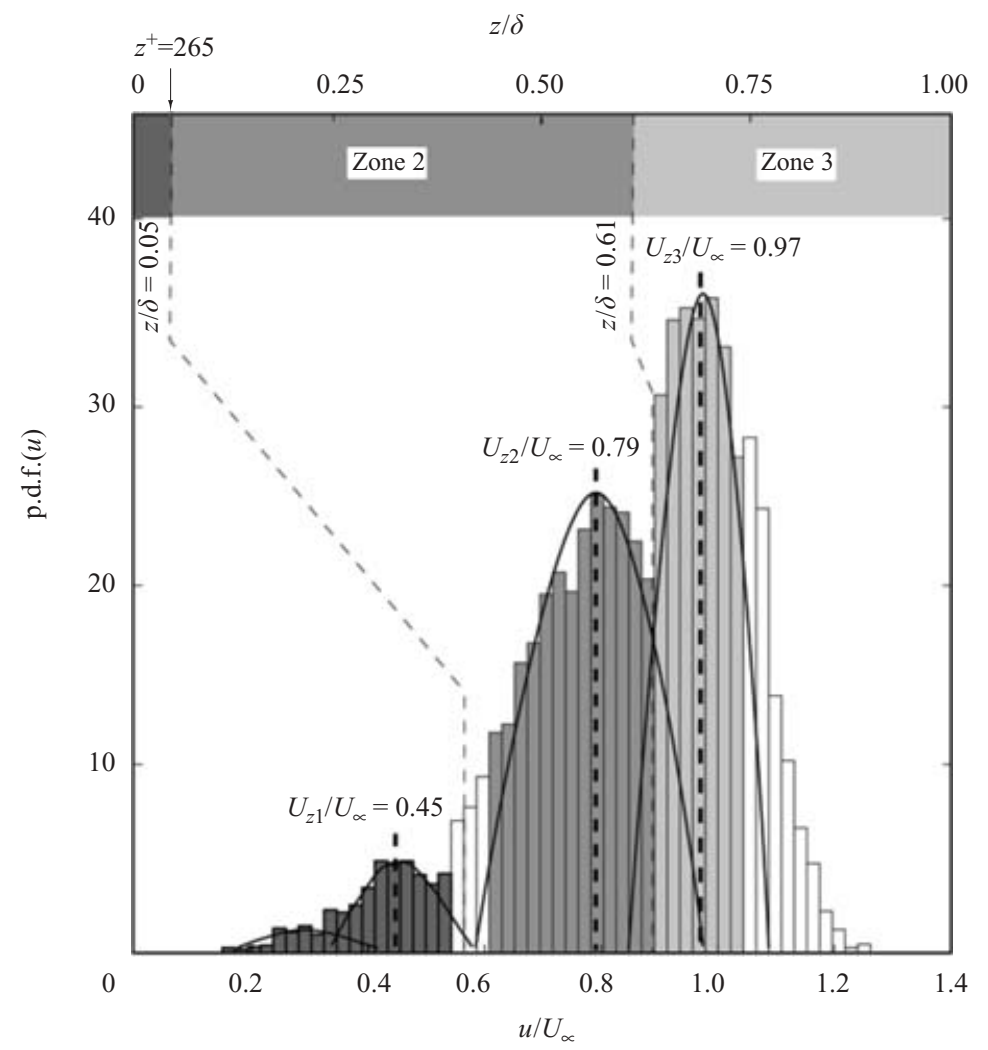

FIGURE 4. Histogram of the relative instantaneous velocity component $u$ for run A.

(mean length, mean inclination, polymodal p.d.f.s) are similar to those found in the free-stream experiments of AMT00, suggesting the validity of the outer-flow organization concept based on the presence of hairpin packets.

\subsection{Large coherent shear stress structures (LC3S)}

The quadrant threshold technique proposed by Lu \& Willmarth (1973) was originally developed for the study of the bursting phenomenon in the buffer region of shear flows. It was first applied to hot-film measurements and more recently to laser Doppler anemometer (LDA) and ADV measurements (Bogard \& Tiedermann 1986; Séchet \& LeGuennec 1999; López \& Garcia 1996; Nikora \& Goring 2000). Newly developed measuring tools such as PIV or ADVP offer the possibility of computing mean quadrant threshold results and analysing conditionally sampled quasi-instantaneous shear stress structures in the $(x, z)$-plane. Compared to conventional quadrant threshold analysis based on single-point measurements, this approach provides additional information on shear structure organization. Therefore we define the dimensionless momentum flux variable $\gamma(z, t)=u^{\prime} w^{\prime} / \overline{u^{\prime} w^{\prime}}$ sampled as a function of two conditions:

(i) The threshold value $H$ which selects instantaneous events of amplitude $|\gamma| \geqslant H$. At a given depth, a constant value of the threshold $H$ defines a hyperbolic curve of amplitude $H \overline{u^{\prime} w^{\prime}}$ in the $\left(u^{\prime}, w^{\prime}\right)$-plane. The unselected events of amplitude $|\gamma|<H$ are called hole events.

(ii) The quadrant $q$ of $\gamma(z, t)$ in the $\left(u^{\prime}, w^{\prime}\right)$-plane. Four event types called outward interactions $\left(u^{\prime}>0, w^{\prime}>0\right)$, ejections $\left(u^{\prime}<0, w^{\prime}>0\right)$, inward interactions $\left(u^{\prime}<0\right.$, 


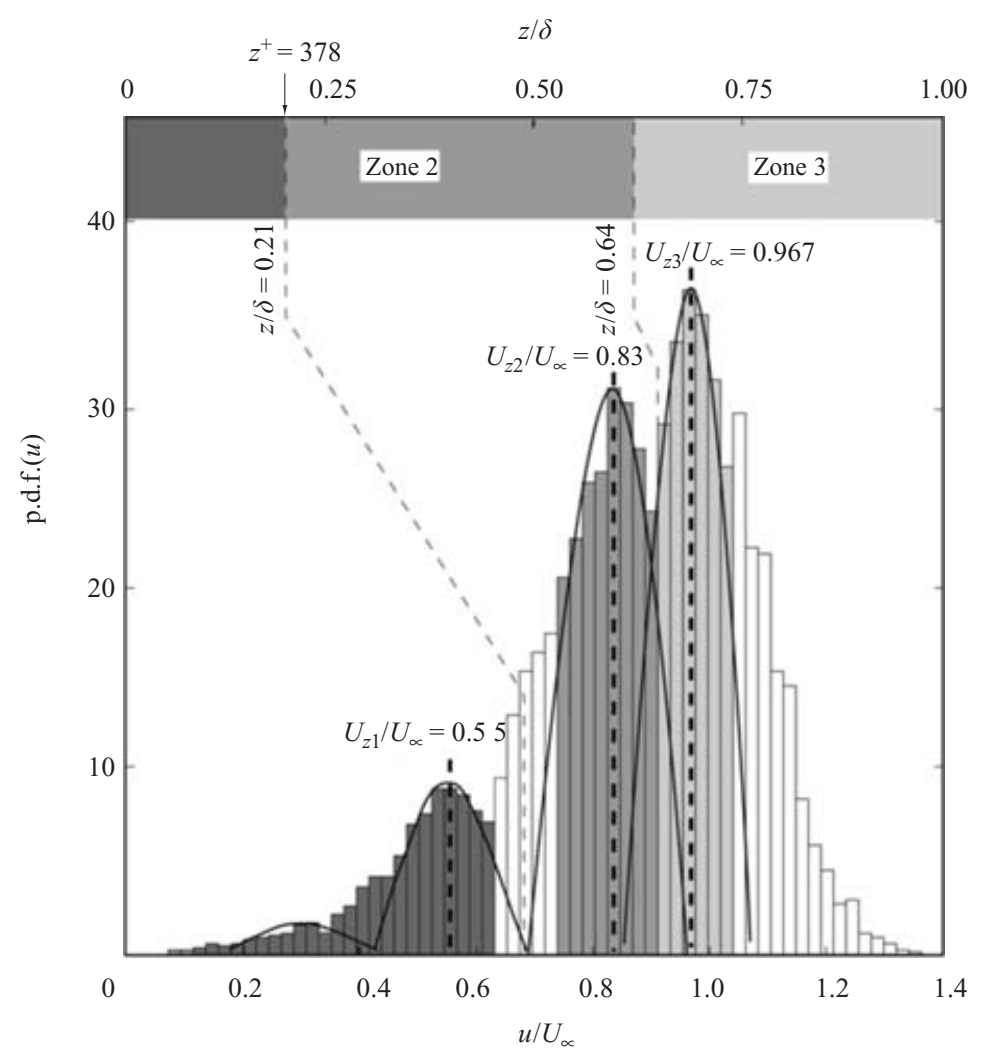

FIGURE 5. Histogram of the relative instantaneous velocity component $u$ for run B.

$\left.w^{\prime}<0\right)$, and sweeps $\left(u^{\prime}>0, w^{\prime}<0\right)$, are related to the four quadrants of the $(x, z)$ plane. Figures 6 and 7 show the application of this conditional sampling method to the instantaneous flow field.

The grey zones in figures $6(a)$ and $7(a)$ represent the zones of uniform momentum with linear approximations of the separation contours between Zones 2 and 3 (dashdotted lines). The streamwise distance in the upper traces is equal to $10 \delta$ and $5 \delta$, for runs A and B respectively. The barred and grey 'sanded' patches in figures 6 and 7 represent regions of ejections and sweeps sampled with a threshold value of $H=2$.

The large selected shear stress patches denoted by closed contours indicate that vertical coherence extends over most of the outer-flow region. Examples are the ejection and sweep events located at $x / \delta=4.25$ and $x / \delta=6.8$ in figure 6 , respectively or the ejection and sweep events located at $x / \delta=3.75$ and $x / \delta=2$ in figure 7 , respectively. Compared to the vertical coherence distance, the streamwise coherence of the shear stress structures is seen to be smaller by a factor of 2 to 4 . Examples are the patches located at $x / \delta=1$ and $x / \delta=0.2$ in figure 6 or at $x / \delta=2$ and $x / \delta=1.25$ in figure 7. Owing to the distinct outer-flow dimensions of these momentum flux events, we call them large-scale shear stress structures (LC3S). They are clearly identifiable with closed contour lines for a threshold value above $H \approx 2$. This threshold value is approximately half the burst detecting value (Bogard \& Tiederman 1986). This is not surprising since the pseudo-cyclic ejection/sweep sequence linked to the bursting phenomenon is a buffer layer process involving inner scales which are smaller by an 


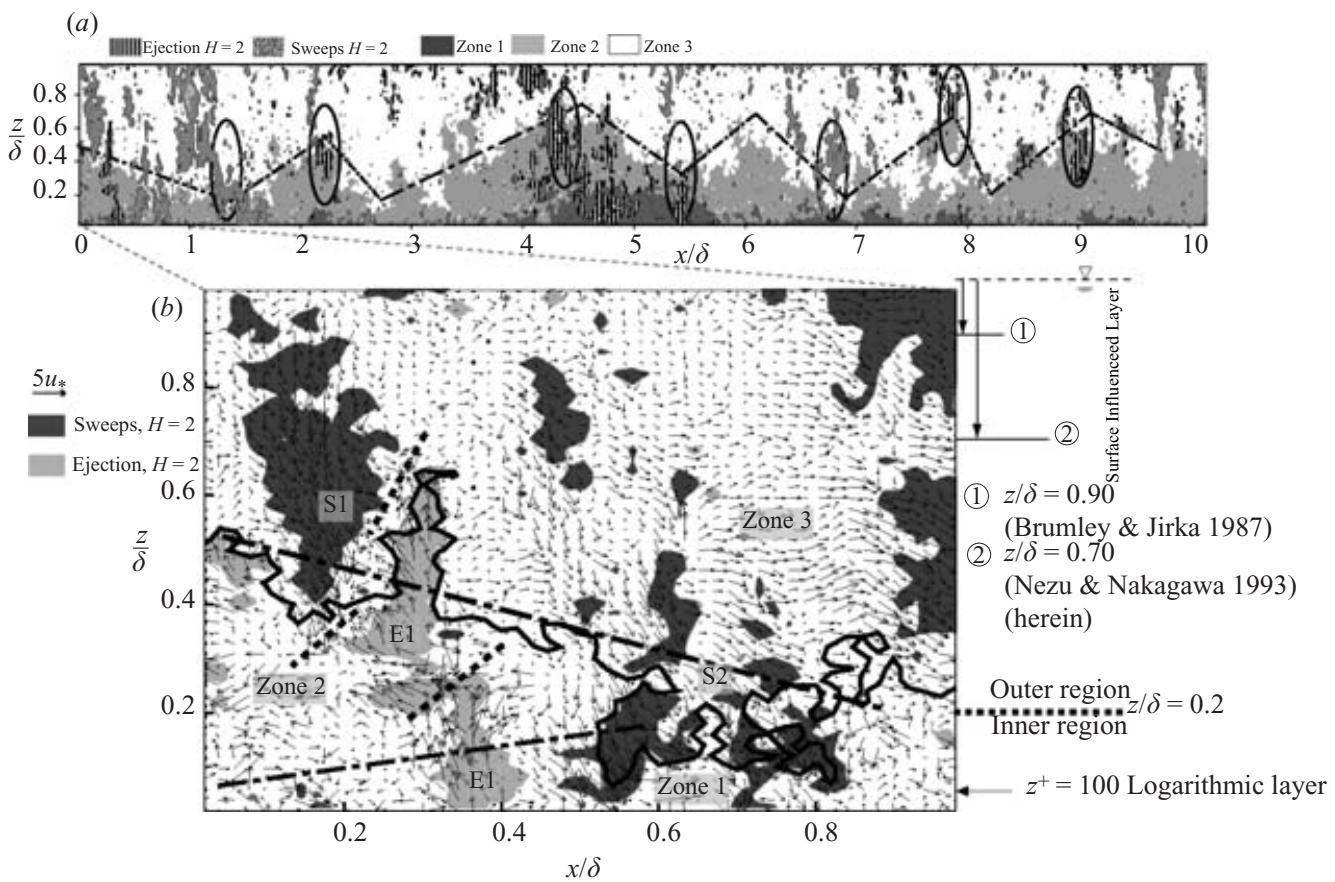

FiguRE 6. Flow organization into uniform zones of streamwise momentum (see figure 3 caption for definitions of zones) Large coherent shear stress structures (LC3S) for $H=2$, fitted separation contours between Zones 2 and 3, stagnation zones between ejections and sweeps (run A). (b) An enlargement of the left-hand part of $(a)$.

order of magnitude than the outer-scale properties of zones of uniform momentum discussed in the previous two sections.

Figures $6(a)$ and $7(a)$ also reveal that the spacing between consecutive LC3S matches fairly well the wavelength of the pseudo-cyclic recurrence found for Zones 2 and 3 in figure 3. For both runs we observe similar patterns of LC3S and the pseudoperiodic variation between sequences of uptilted and downtilted contour lines between Zones 2 and 3. The ellipses in figures $6(a)$ and 7(a) depict ejection- and sweep-type LC3S located at the maxima and minima of the fitted separation lines, respectively. This coincidence supports the idea of a strong link between the location and type of LC3S with the location and mean inclination of the zones of uniform momentum. AMT00's data show uptilted separation lines with similar geometrical characteristics in terms of mean inclination and streamwise length. The downtilted separation lines seen herein are rare and have much shorter dimensions in the streamwise direction. We have not found such systematic up/down sequences in the literature.

Figures $6(b)$ and $7(b)$ show a close-up of the two-dimensional turbulent velocity field over a streamwise distance of $1 \delta$. The stagnation regions between two adjacent ejection and sweep events are marked by dashed lines. Whether the detected stagnation regions correspond to the necks of individual hairpin vortices is still uncertain at present owing to the lack of spatial resolution for direct vorticity and/or swirling strength calculations. Nevertheless it can be seen that the growth angles of the individual stagnation regions increase with distance from the bed. For example, the inclinations of the two stagnation regions in Zone 2 of figure 6 are similar (around $45^{\circ}$ ), and 


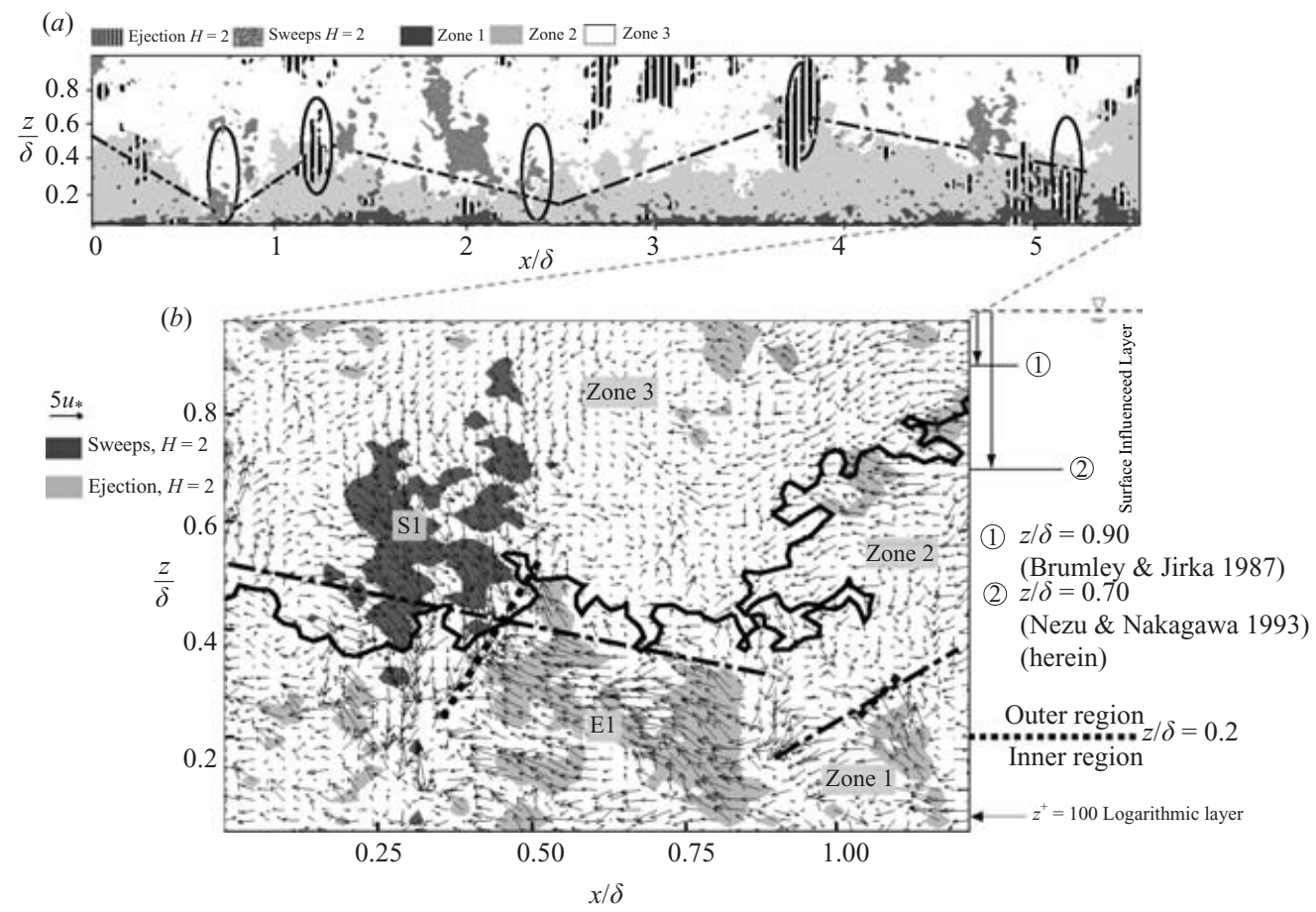

FIGURE 7. Flow organization into uniform zones of streamwise momentum (see figure 3 caption for definitions of zones) large coherent shear stress structures (LC3S) for $H=2$, fitted separation contours between Zones 2 and 3, stagnation zones between ejections and sweeps (run B). (b) An enlargement of the right-hand part of $(a)$.

both are smaller than the inclination of the stagnation region in Zone 3, which is close to $80^{\circ}$. However, the uptilted and downtilted angles of the fitted separation lines (dash-dotted lines) are much smaller (about $18^{\circ}$ ) and nearly constant with depth as seen in figures $6(a)$ and $7(a)$.

In order to determine whether the observed LC3S modify the mean quadrant threshold results in open-channel flows, we compare them to well-documented literature results. We define the quantity $R S_{q}(z, H)$ corresponding to the time-averaged momentum flux for $\gamma(z, t)$ in quadrant $q$ and $|\gamma(z, t)| \geqslant H$. Two estimates will be compared:

(i) a direct estimate from the velocity measurements:

$$
\begin{aligned}
R S_{q}(z, H)=T^{-1} \int_{0}^{T} \gamma(z, t) D_{1}[\gamma(z, t)] \mathrm{d} t & \text { with } D_{1}[\gamma(z, t)] \\
& \begin{cases}=1 & \text { for }|\gamma| \geqslant H \text { and } \gamma \text { in } q \\
=0 & \text { otherwise }\end{cases}
\end{aligned}
$$

(ii) the estimate from the first-order moment of the theoretical probability density function $p_{q}[\gamma(z, t)]$ :

$$
R S_{q}(z, H)=\int_{H}^{\infty} \gamma(z, t) p_{q}[\gamma(z, t)] \mathrm{d} \gamma
$$



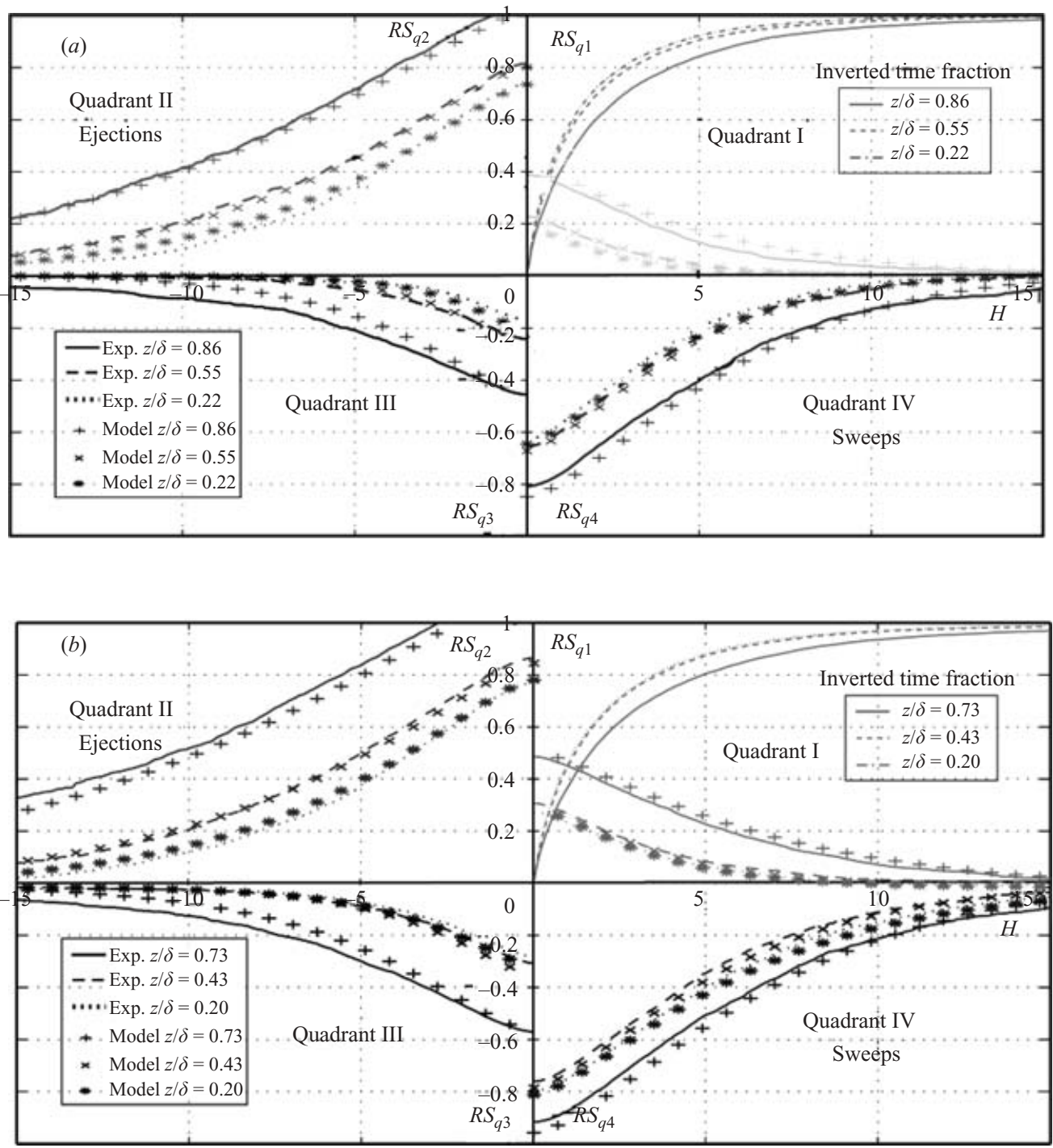

FiguRE 8. Quadrant threshold distribution for runs $(a) \mathrm{A}$ and $(b) \mathrm{B}$.

Equation (1) represents the quadrant threshold technique proposed by Lu \& Willmarth (1973). The second method, (2), requires a theoretical expression for the probability density function of $\gamma(z, t)$ in quadrant $q$. We use a cumulant discard expansion series of the characteristic functions of $u^{\prime} /\left(\overline{u^{\prime 2}}\right)^{1 / 2}$ and $w^{\prime} /\left(\overline{w^{\prime 2}}\right)^{1 / 2}$ limited to the third order. Since both components have weakly non-Gaussian statistical properties, classic Gram-Charlier functions are used. Over a wide range of flow conditions these functions have given good results in previous studies such as Antonia \& Atkinson (1973), Nakagawa \& Nezu (1977), Raupach (1981), López \& Garcia (1999), Hurther \& Lemmin (2000), and Fer, McPhee \& Sirevaag (2004).

In figures $8(a)$ and $8(b)$, the symbols and lines correspond to the results of the direct (equation (1)) and theoretical (equation (2)) estimates, respectively, for $R S_{q}(z, H)$ at three different depths in the outer regions for runs $\mathrm{A}$ and $\mathrm{B}$, respectively. The typical dominance of ejections and sweeps is found for the theoretical and experimental 
estimates of the fractional momentum flux. These results are in good agreement with literature results on quadrant distributions in open-channel flows (Nakagawa \& Nezu 1977; López \& Garcia 1996; Hurther \& Lemmin 2000). The use of a third-order Gram-Charlier function for the estimate of the higher-order terms of the p.d.f.s results in small discrepancies between the theoretical and the experimental curves. Another typical characteristic of ejection and sweep distributions is their much longer tail in $H$ compared to Quadrant I and III events. This observation combined with the time fraction distributions of the hole events at three different depths (represented by the three curves increasing with $H$, in Quadrant I) reveals that an important fraction of the total shear stress results from intense (high amplitudes during short time periods) momentum flux events. This confirms the representations of the LC3S shown in figures 6 and 7. The trend of all curves for run B is found to be very similar to the results of run A. The mean shear structure of the outer layer may be considered common for all Reynolds numbers and bed roughnesses, which supports the idea of a universal flow organization concept for the outer region. It can also be seen that the tail of sweeps vanishes earlier in $H$ than in the case of ejections. As a consequence, ejections and sweeps do not present symmetrical contributions (Raupach 1981). This aspect and its consequence for the TKE budget will be analysed in detail in the following sections.

Figures $8(a)$ and $8(b)$ exhibit the typical non-uniform quadrant distribution found in the literature for shear boundary layers (Nakagawa \& Nezu 1977; Raupach 1981; Katul et al. 1994; López \& Garcia 1996; Hurther \& Lemmin 2000) suggesting that the presence of LC3S in the outer region is not specific to the present experiments but is common to shear flows. Furthermore the values of $R S_{4}(z, 0)-R S_{4}(z, 2)$ and $R S_{2}(z, 0)-R S_{2}(z, 2)$ vary between $10 \%$ and $20 \%$, respectively, for both runs. As mentioned earlier, LC3S are shown by closed contours for $H \approx 2$ indicating that they are dominant contributors to the mean shear stress in the outer layer. Finally, the present mean shear results are also in good agreement with previously mentioned shear boundary layer studies. The importance of the present study is that it has succeeded in identifying and characterizing LC3S that are associated with the zonal momentum organization of the shear boundary layer.

\section{Turbulent transport of turbulent kinetic energy (TKE) in the outer region}

The previous sections addressed the existence of LC3S and their strong association with uniform regions of streamwise momentum. The representations of LC3S in figures 6 and 7 combined with the quadrant threshold results in figures $8(a)$ and $8(b)$ showed that LC3S are dominant contributors to mean shear stress in the outer region. As seen in figures 6 and 7 the vertical extent of LC3S can cover important fractions of the outer region. This vertical large-scale dimension supports a direct link in turbulent momentum flux between the inner region and the SIL (surface-influenced layer). The following sections will quantify whether this link is relevant for TKE transport through the outer region and its role in the TKE budget in the SIL.

The profiles of normalized shear production $P \delta / u_{*}^{3}$ and TKE dissipation rate $-\varepsilon \delta / u_{*}^{3}$ are presented in figure $9(a)$ for run C. $P=-\overline{u^{\prime} w^{\prime}} \partial_{z} \bar{u}$ denotes the work done by the turbulent shear stress $-\rho \overline{u^{\prime} w^{\prime}}$ against the mean deformation of the flow. For 2D mean flow conditions no other mechanism produces TKE except in the vicinity of the bed where molecular effects cannot be neglected. However the roughness layers for runs $\mathrm{A}, \mathrm{B}$, and $\mathrm{C}$ are larger than the viscous sublayers and therefore viscous effects are absent. In the following the TKE dissipation is estimated from the turbulence 

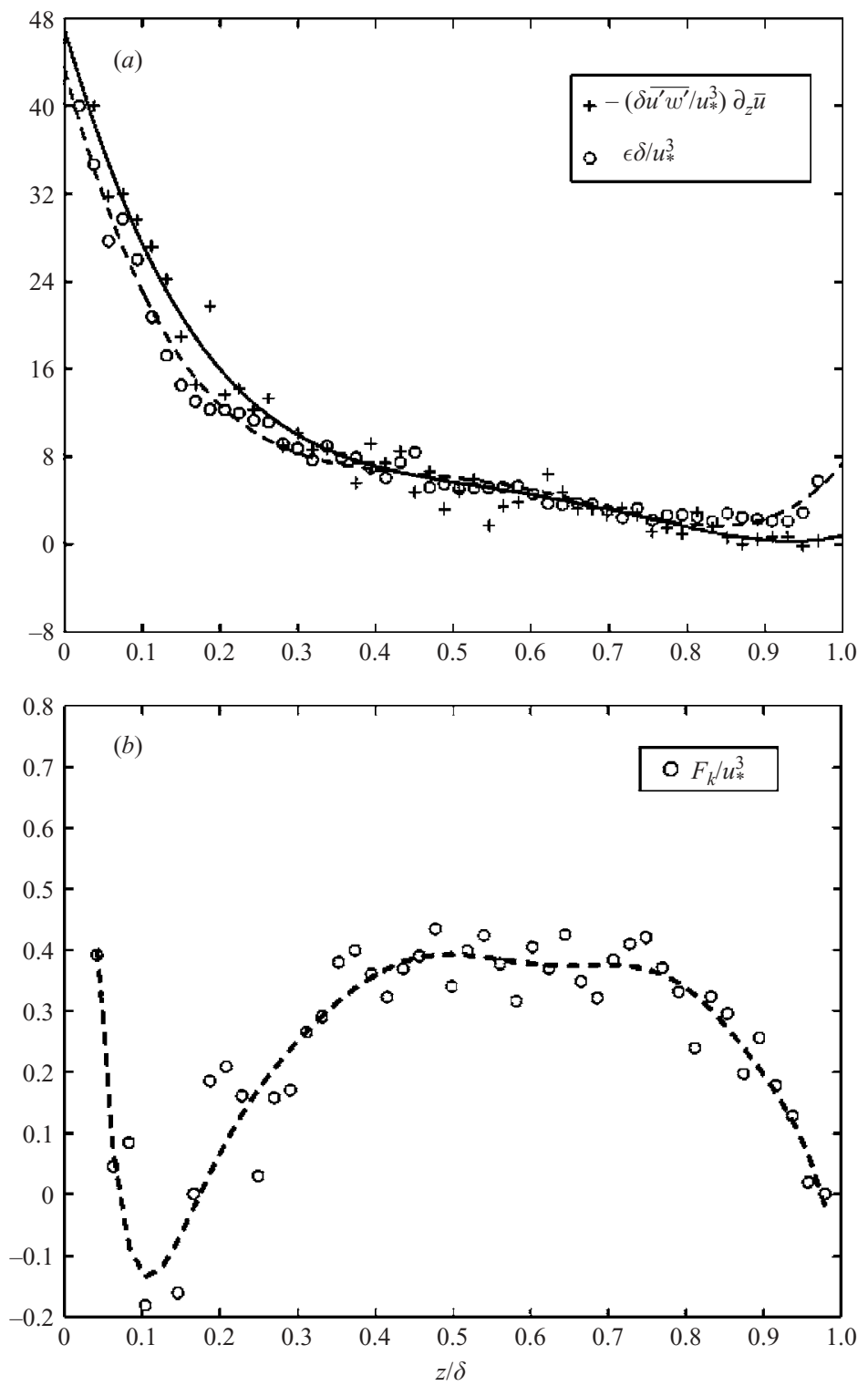

FIgURE 9. (a) Profile of measured and fitted shear production and dissipation for run C. (b) Profile of measured and fitted TKE flux for run C.

spectrum of the streamwise component after applying the Doppler noise reduction method described in Hurther \& Lemmin (2001) and Blanckaert \& Lemmin (2005).

For $z / \delta<0.2$, the maximum dissipation is seen to be smaller than the production, which results in a residual of TKE. For $0.25<z / \delta<0.75$ (called the intermediate region after Nezu \& Nakagawa 1993), a local equilibrium between production and dissipation is observed. In the SIL for $z / \delta>0.75$, TKE dissipation exceeds the production. It should be noted that the outer region of an open-channel flow is divided into the intermediate region and the SIL.

Figure $9(b)$ shows the mean TKE flux for run $C$ given by $F_{k}=0.5\left(\overline{u^{\prime 2} w^{\prime}}+\overline{v^{\prime 2} w^{\prime}}+\overline{w^{\prime 3}}\right)$ and normalized by $u_{*}^{3}$. In the inner region the difference between the shear production 
and the dissipation rate (see figure $9 a$ ) is the most probable source of the TKE flux increasing with depth. The TKE flux is nearly constant between $0.35<z / \delta<0.75$ (figure $9 b$ ), inducing a negligible transport term $-\partial_{z}\left(F_{k}\right)$. This coincides fairly well with the region of local balance between production and dissipation seen in figure $9(a)$. In the SIL, the TKE flux decreases at a nearly constant rate for $0.75<z / \delta<0.9$, whereas the dissipation rate is almost constant for $0.75<z / \delta<0.9$ (for the non-fitted circles in figure $9 a$ ) and increases for $z / \delta>0.9$. The shear production is negligible above $z / \delta=0.8$. This suggests that the TKE transported through the intermediate region into the SIL can act as a local source of turbulence dissipation, since the mean shear does not work against a mean flow deformation in the SIL. Furthermore, the spatial decay rate of the TKE flux increases close to the free surface at $z / \delta=0.95$. This increase is also found in figure $9(a)$ for the dissipation rate at approximately the same depth. The observed local balance for $z / \delta>0.2$ between the spatial rate of TKE flux and the difference between shear production and dissipation rate implies that the mean transport by pressure diffusion is negligible in the outer-flow region. This finding agrees well with DNS results in the outer region of an open-channel flow obtained by Handler et al. (1993). They showed that TKE transport is nearly constant in the SIL and that the transport of turbulent energy by pressure diffusion is negligible except in the vicinity of the free surface $(z / \delta>0.95)$ where the simulation predicts an increase of the pressure diffusion term. This range is not resolved in our case. Based on the previous results for run $\mathrm{C}$ and a comparison with DNS (in smooth-wall turbulent flows with low Reynolds numbers), we observed that TKE is transported at a local equilibrium through the intermediate region into the SIL where its local spatial decay rate agrees well with the local TKE dissipation rate. The agreement between the results of Handler et al. (1993) and those presented here suggests a negligible effect of roughness on the TKE budget in the outer region.

The above analysis of the mean quantities has revealed the crucial role played by the turbulent transport of TKE through the outer region, particularly its capacity to produce TKE dissipation in the SIL. In order to quantify the contribution of LC3S to this TKE flux in the outer region, we apply the following quadrant threshold method to the instantaneous TKE flux:

$$
\begin{aligned}
& T F(H)=\left(F_{k} T\right)^{-1} \int_{0}^{T} F_{k}(t) D_{\text {hole }}\left[u^{\prime} w^{\prime}(t)\right] \mathrm{d} t \\
& \text { with } \\
& D_{\text {hole }}=\left\{\begin{array}{ll}
1 & \text { if }|\gamma|<H \\
0 & \text { otherwise, }
\end{array}\right\}
\end{aligned}
$$

where $D_{\text {hole }}$ is a weighting function selecting instantaneous TKE flux events for which the instantaneous momentum flux amplitude is less than $H$ times the local mean shear stress. These events are commonly called hole events. $F_{k}$ is the mean TKE flux at a given depth. The conditionally sampled TKE flux will be compared to the typical quadrant threshold quantities given in equation (1) and those selected with the weighting function $D_{\text {hole }}$ used in equation (3). The quantities $R S_{q}$ and $T F_{k}$ are therefore normalized by the local mean shear and TKE flux.

This method will be applied to all runs in this study, as well as to the AMT00 data, available at: http://ltcf.tam.uiuc.edu/Downloads/Data/BL/index.html.

Since the transversal velocity component was not measured in AMT00, we will approximate their instantaneous TKE flux by $F_{k}=0.75\left(\overline{u^{\prime 2} w^{\prime}}+\overline{w^{\prime 3}}\right)$. This approximation gave good results in previous studies (Raupach 1981; Nezu \& Nakagawa 1993). 

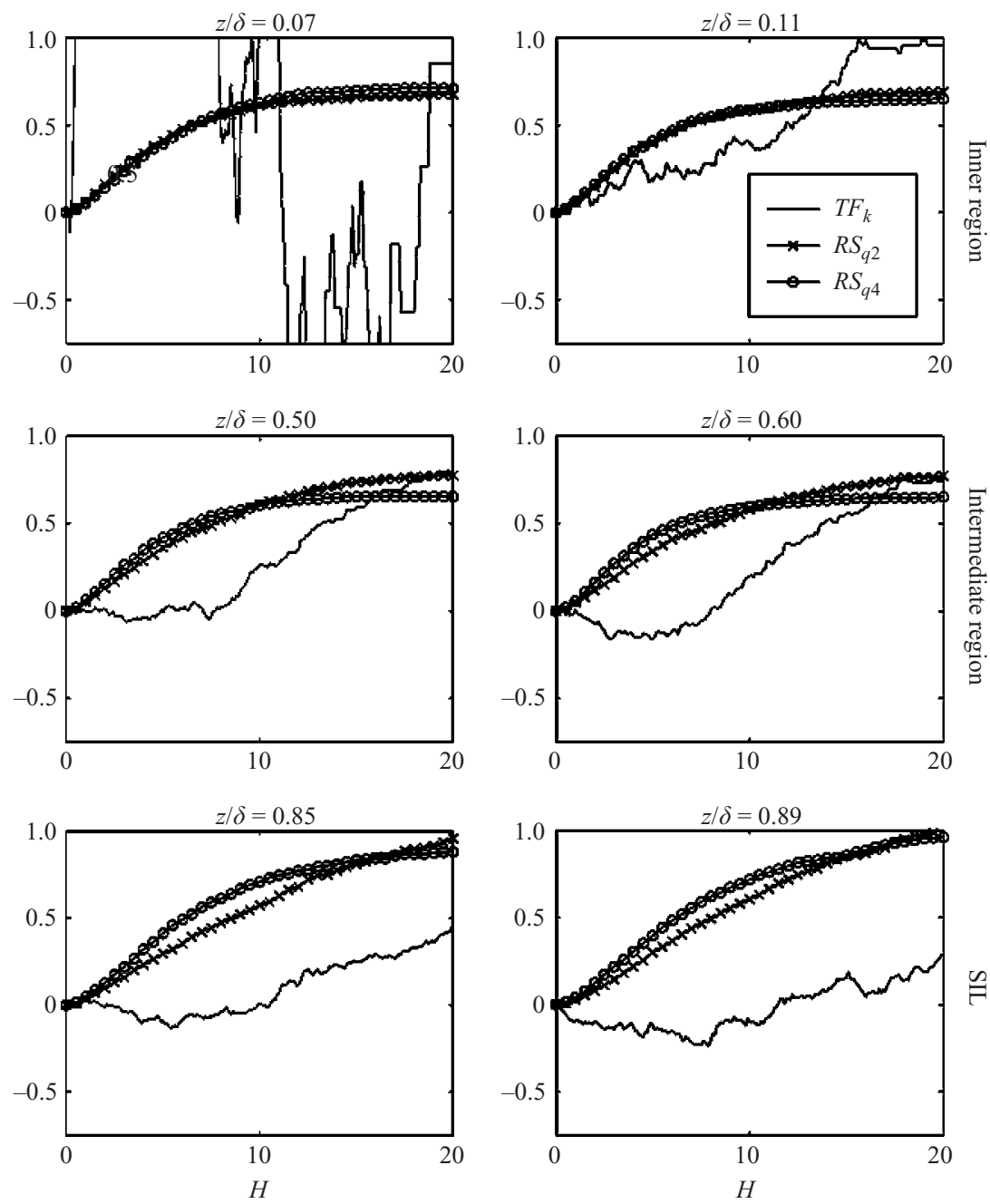

FIgURE 10. Conditionally sampled $T F_{k}, R S_{2}$ and $R S_{4}$ for six different depths for run A.

Figures 10 to 14 show the conditionally sampled shear stress for Quadrants II (ejections) and IV (sweeps), and the TKE flux as a function of $H$ for runs A, B, C, $\mathrm{AH}$ and $\mathrm{AL}$, respectively. In each figure we present results at two depths in the inner region and four depths in the outer region (two in the intermediate and two in the SIL). It should be noted that the SIL does not exist for runs AH and AL, since the experiments were conducted in a free-stream shear boundary layer. For comparison, results in figures 10 to 14 are shown for normalized depth, where the differences in normalized flow depths between the runs never exceed $2 \%$.

We observe good agreement between the $R S_{2}$ and the $R S_{4}$ curves for all runs at comparable depths. All $R S_{2}$ and $R S_{4}$ curves monotonically increase until they reach a plateau. In the inner region the $H$ value at which the plateau begins is the same for $R S_{2}$ and $R S_{4}$, except for run AL which shows slight differences between $R S_{2}$ and $R S_{4}$. In the outer region, sweeps reach their plateau earlier than ejections thus confirming the higher contribution of ejections compared to sweeps (figure 4). The $R S_{q}$ curves shown in figures 10 to 14 are identical to those in figures $8(a)$ and $8(b)$, 

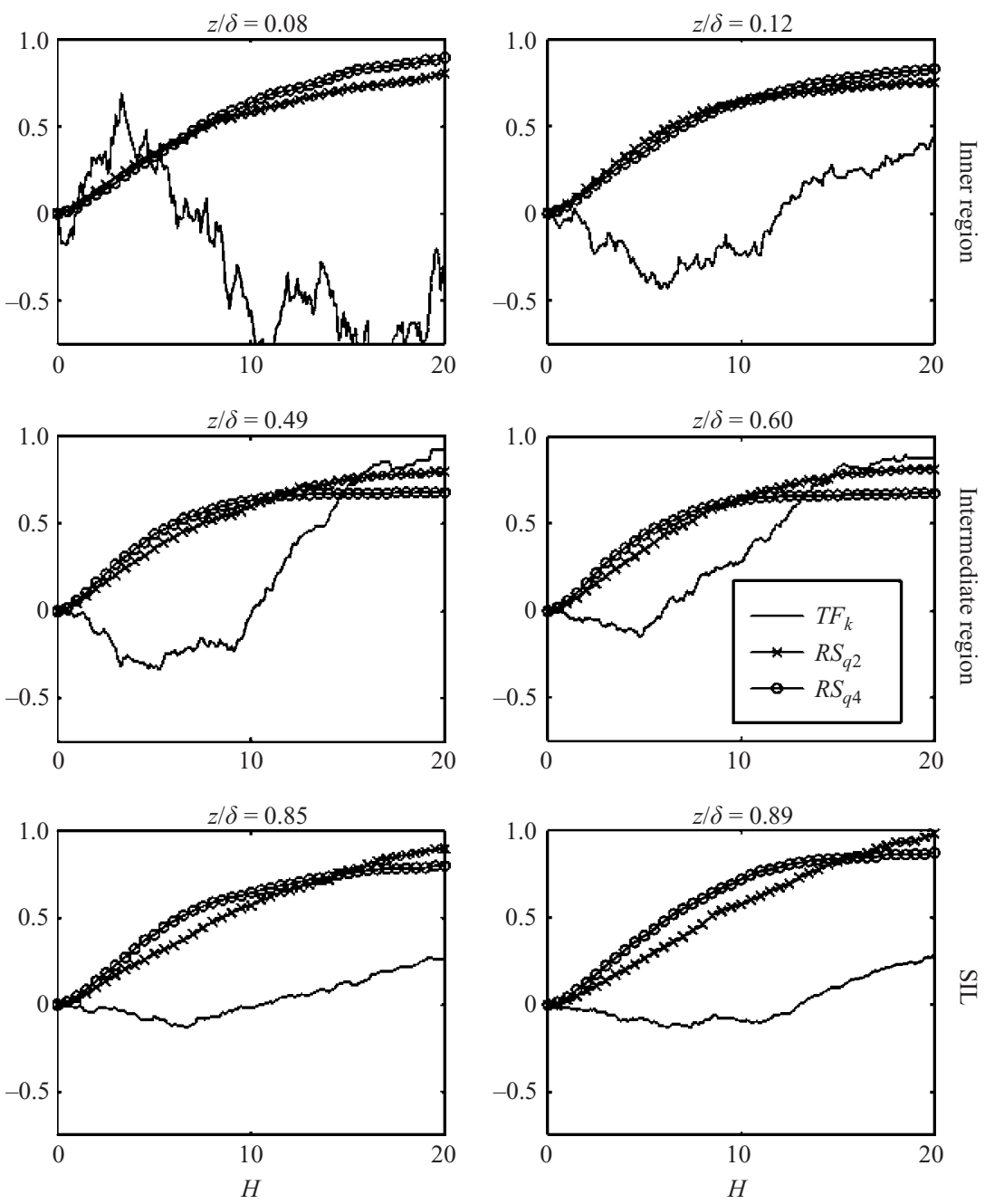

Figure 11. Conditionally sampled $T F_{k}, R S_{2}$ and $R S_{4}$ for six different depths for run B.

but have a different weighting function $D_{\text {hole. }}$. The good agreement of $R S_{q}$ seen in figures 10 to 14 for all runs emphasizes the robustness of the quadrant threshold results in free-surface and free-stream shear boundary layers. It also supports the earlier finding of the dominant contribution of LC3S to shear stress for $H$ above 2, since the difference in $R S_{q}$ between $H=0$ and $H=2$ is less than $20 \%$.

In the inner region the $T F_{k}$ curves differ between individual runs and from the $R S_{q}$ curves. The high variability in $T F_{k}$ and the noise-like signal structure shows that the TKE flux is not controlled by $u^{\prime} w^{\prime}$ structures in the inner region. In the intermediate region, however, we can see that at comparable depths the trends of $T F_{k}$ are similar for all runs. In particular, we find that more than $90 \%$ of the TKE flux is produced by $u^{\prime} w^{\prime}$ momentum flux events in the interval $5<H<20$. For $H<5$ all the curves show a weak absolute contribution to $T F_{k}$ by $u^{\prime} w^{\prime}$ events $(20 \%$ at most for run $\mathrm{AH})$. In the SIL, good consistency of the curves is seen between the different runs but only about $50 \%$ of $T F_{k}$ is reached in the interval $5<H<20$. 

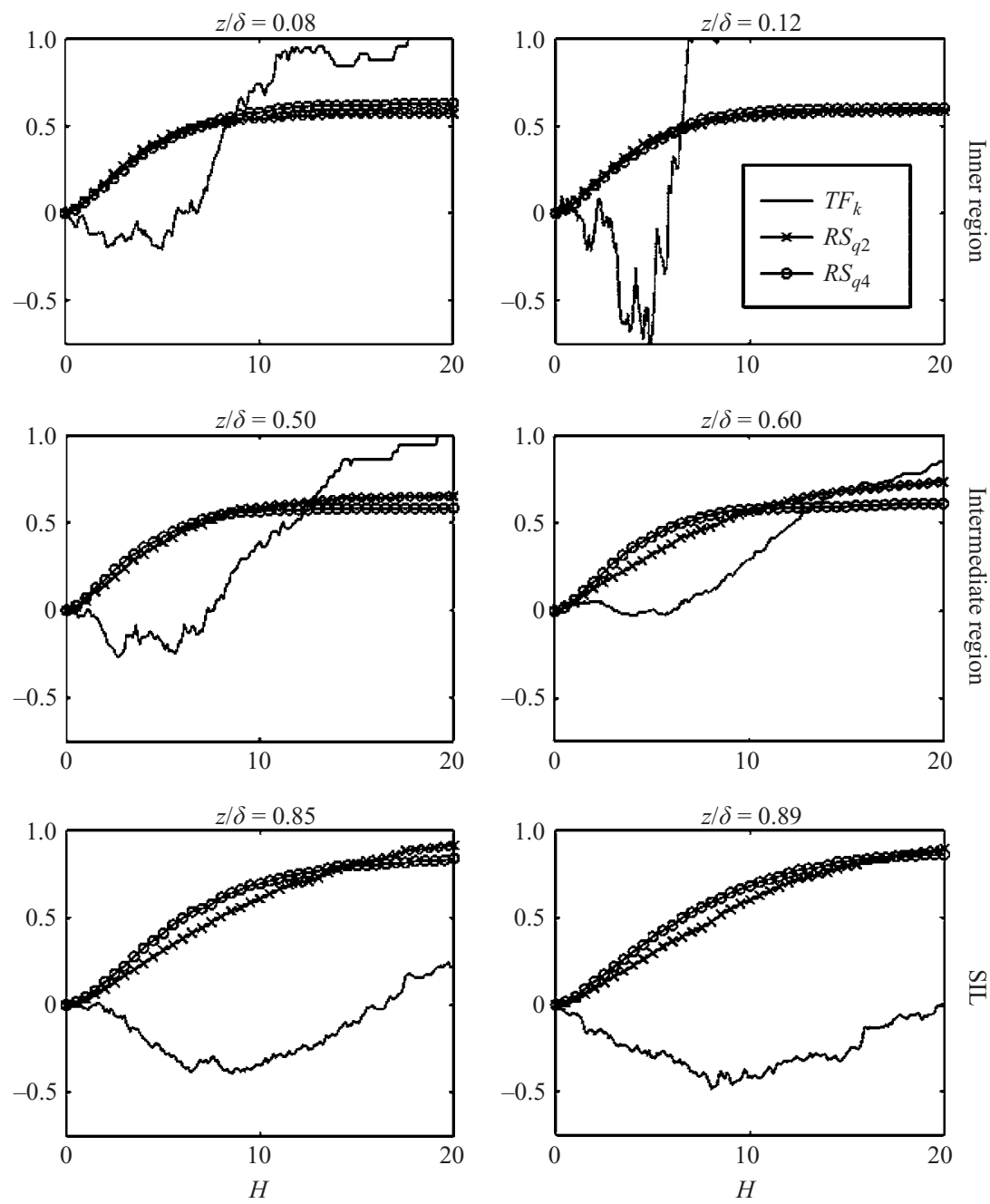

FIgURE 12. Conditionally sampled $T F_{k}, R S_{2}$ and $R S_{4}$ for six different depths for run C.

Figures 10 to 14 show that in the intermediate region the TKE flux is almost entirely driven by high- $u^{\prime} w^{\prime}$-momentum flux events in the interval $5<H<20$. The weak $u^{\prime} w^{\prime}$ contribution by Quadrant I and III events in this interval (shown in figures $8 a$ and $8 b$ ), suggests that LC3S play an important role in the generation of the vertical TKE flux. Nevertheless significant differences are found in the trends with $H$ of $T F_{k}$ and $R S_{q}$ curves, particularly in the range $0<H<5$, where $T F_{k}$ often remains negligible, whereas the contribution of $R S_{q}$ increases to about $50 \%$.

\section{LC3S-induced TKE flux}

The significant contribution to the mean TKE flux by $u^{\prime} w^{\prime}$ events in the interval $5<H<20$ was shown in the last section. However the important differences in the trends with $H$ of the $R S_{q}$ and $T F_{k}$ curves have not been elucidated at this stage. In order to understand the role played by LC3S in the generation of mean TKE flux we 

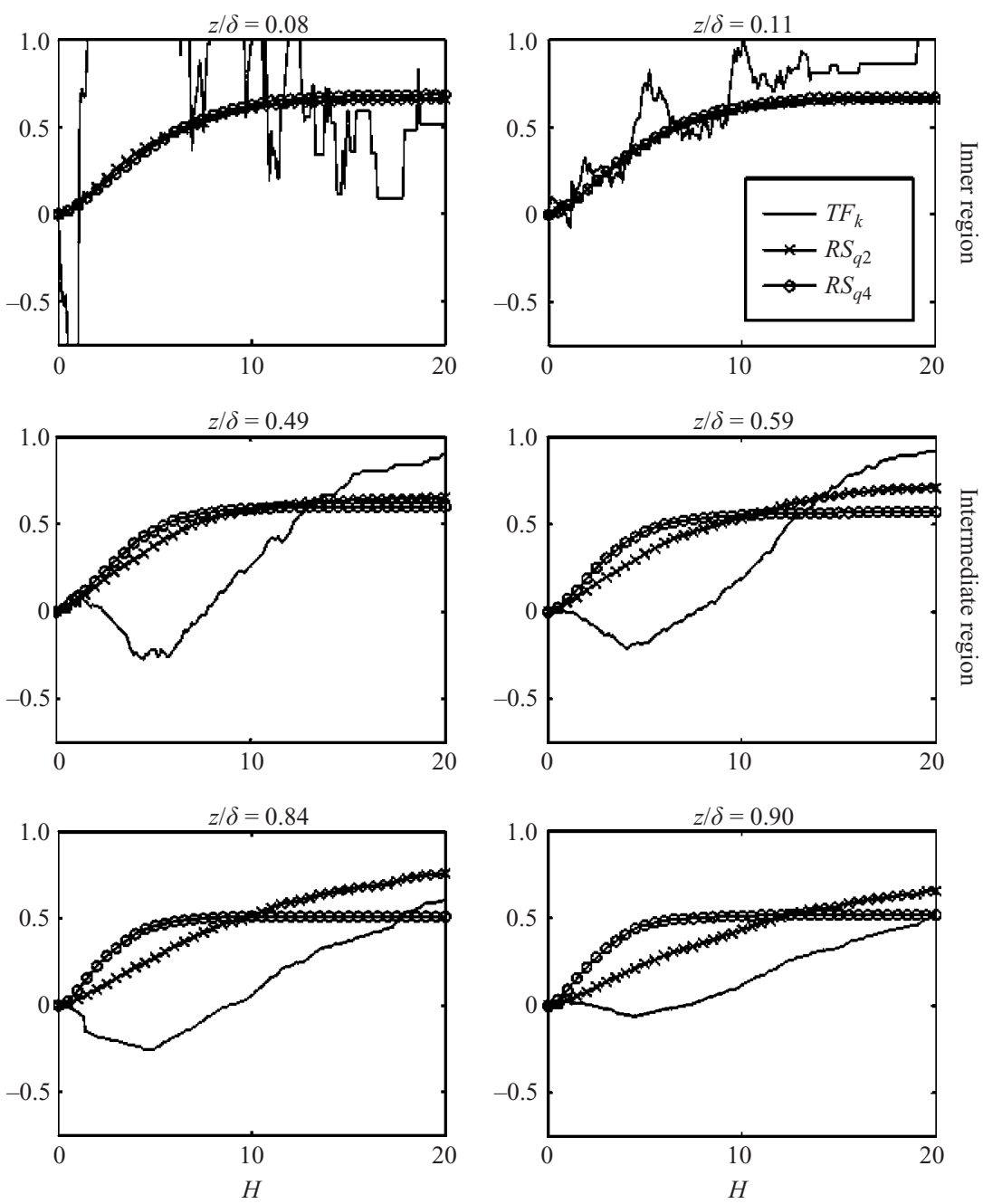

FIGURE 13. Conditionally sampled $T F_{k}, R S_{2}$ and $R S_{4}$ for six different depths for run AH.

propose to evaluate the following parameter:

$$
\Delta R S(H)=R S_{2}(H)-R S_{4}(H)
$$

where $R S_{q}$ are sampled using the weighting function $D_{\text {hole }}$ defined in equation (3). This parameter is a measure for the fraction of the residual momentum flux between ejections and sweeps as a function of the threshold level $H$. The interest in $\Delta R S$ and its plausible connection to $T F_{k}$ is suggested by the fact that the dynamics of the residual energy between production and dissipation such as the TKE flux will most likely depend on the dynamics of a residual of momentum flux given by $\Delta R S$ and not $R S_{q}$. Most of the $R S_{q}$ being produced and contained in LC3S obviously contribute to the production and dissipation of TKE.

Another theoretical link between $T F_{k}$ and $\triangle R S$ is suggested by the equations given by Nakagawa \& Nezu (1977) approximating the conditionally sampled probability density functions of the momentum flux $u^{\prime} w^{\prime}$ in quadrant $q$ by Gram-Charlier functions limited to the third order. It can be seen that zero-valued third-order 

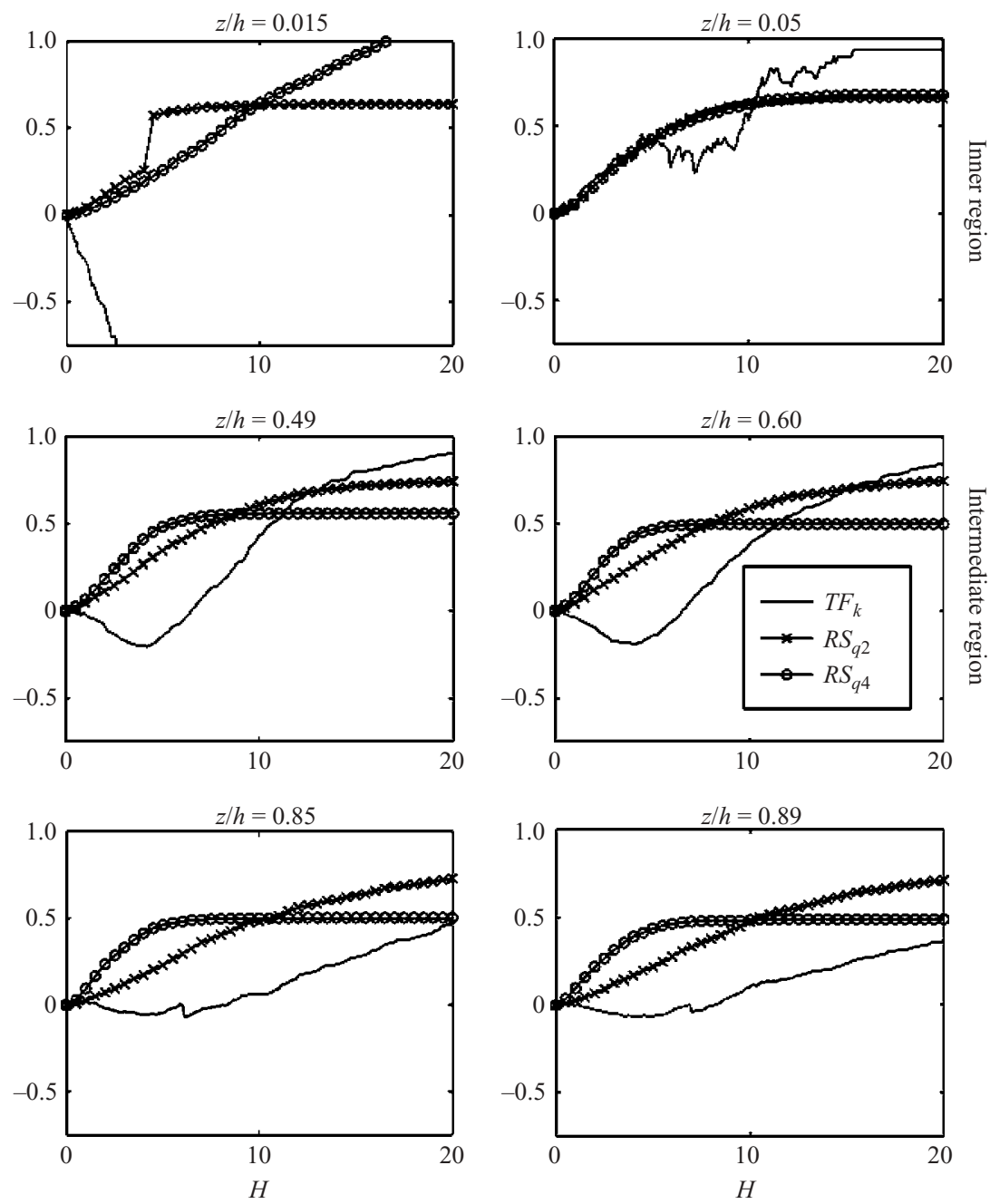

FIGURE 14. Conditionally sampled $T F_{k}, R S_{2}$ and $R S_{4}$ for six different depths for run AL.

moments reduce $\Delta R S$ to zero. The same property is valid for the mean TKE flux, since each of its three terms is proportional to a third-order velocity component. A coupling of the two quantities seems intuitively possible.

In figures 15 to 19 , the $\Delta R S$ curves are compared to the $T F_{k}$ curves. Also shown are the normalized partial derivatives in $H$ of $\triangle R S$ and $T F_{k}$. The third parameter corresponds to $\Delta R S_{D 1}$ evaluated with the weighting function $D_{1}$ in equation (1), for comparison with Raupach (1981) who proposed a linear relation between the mean TKE flux and $\triangle R S$ :

$$
F_{k} \propto \Delta R S_{D 1,0}
$$

where $\Delta R S_{D 1,0}$ is equal to the difference between ejections and sweeps for $H=0$.

In the intermediate region we observe above $H=5$ excellent agreement between the two derivatives for all runs. The curves have a distinct maximum at roughly $H=11$ and a bandwidth in $H$ of around 10. No consistency is seen for the derivatives in the 

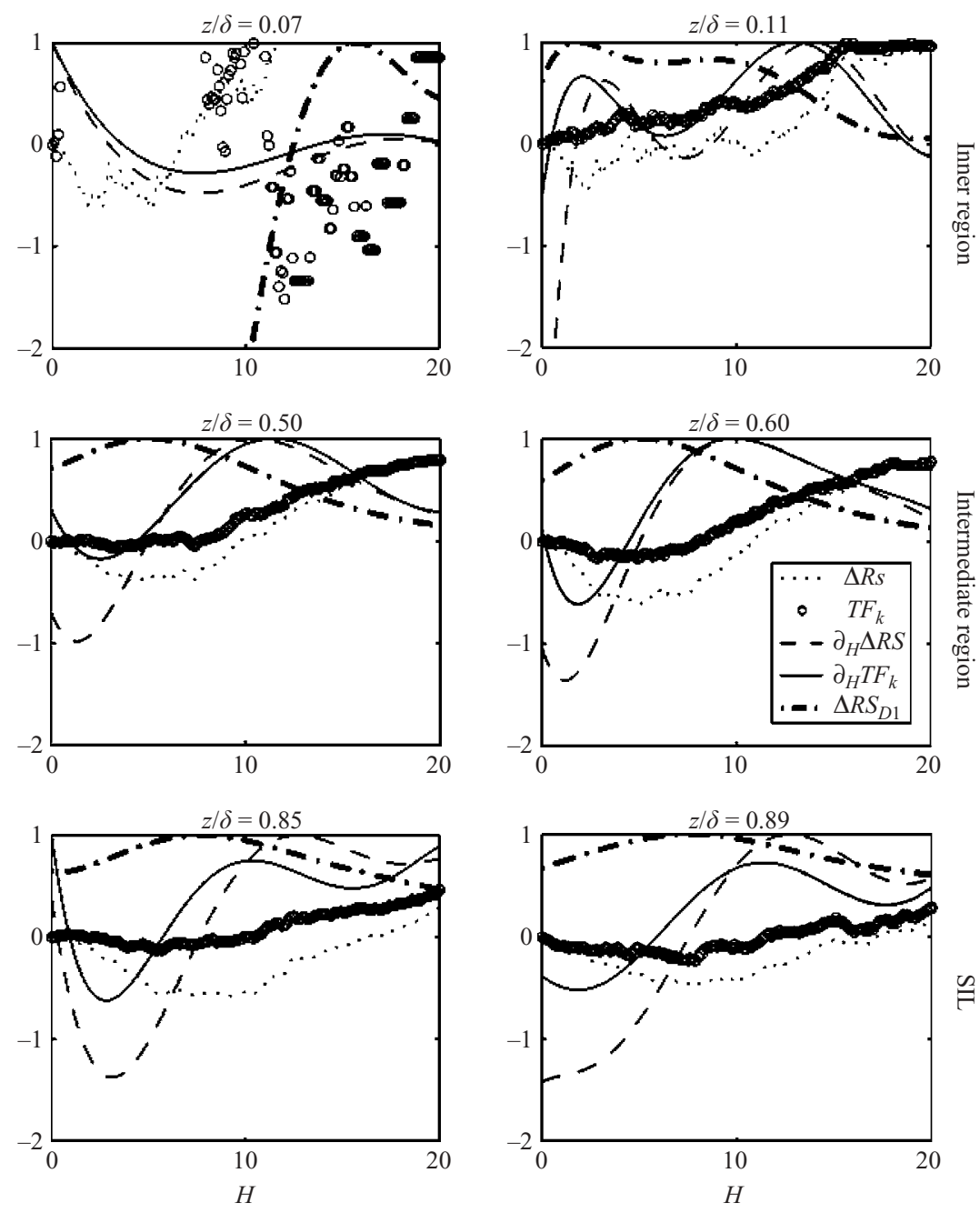

FIGURE 15. Conditionally sampled $\Delta R S, T F_{k}, \partial_{H} \Delta R S, \partial_{H} T F_{k}, \Delta R S_{D 1}$ for six different depths for run $\mathrm{A}$.

inner region. In the SIL, few derivatives agree with each other (run B). However the trends significantly change with depth and between runs.

In the outer region (intermediate and SIL) of the boundary layer, all $\triangle R S_{D 1}$ curves are positive and have a distinct maximum around $H_{\max }=5$. Ejections therefore contribute more than sweeps, as literature results confirm. The difference in ascendant shear stress reaches a maximum for clearly identifiable threshold values in all runs. Furthermore none of the $\Delta R S$ parameters include contributions of Quadrant I and III events, which confirms their negligible role in TKE flux generation. Considering the results of $\S \S 2$ and 3 showing that above $H=2$ the shear stress is predominantly generated by LC3S, we deduce that the maximum value in $\Delta R S_{D 1}$ originates from LC3S.

A comparison of $\Delta R S_{D 1}$ with the derivatives in the intermediate region shows that the location of the maxima, called $H_{\max }$, corresponds to the threshold value at which the two derivatives start to coincide. The $H_{\max }$ value is therefore seen to select LC3S 

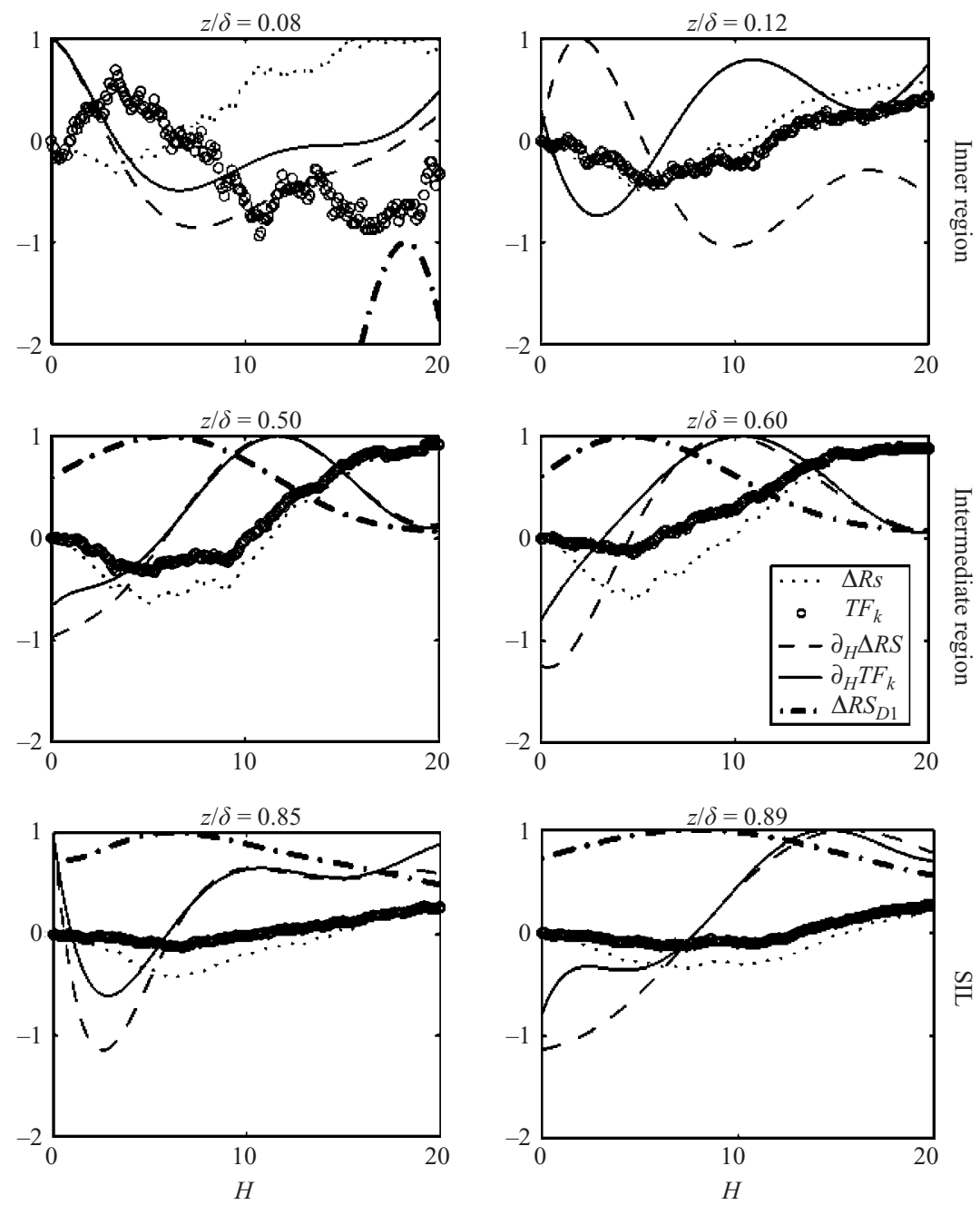

FiguRE 16. Conditionally sampled $\Delta R S, T F_{k}, \partial_{H} \Delta R S, \partial_{H} T F_{k}, \Delta R S_{D 1}$ for six different depths for run $B$.

whose time-averaged net momentum flux (difference between ejections and sweeps) is at the origin of the mean TKE flux.

Raupach's (1981) expression given in equation (5) relates linearly the mean TKE flux to the residual momentum flux at $H=0$. However, as can be seen in figures 15 to 19 , this threshold value does not select structures for which only the difference contributes to TKE flux. For example, in the range $0<H<H_{\max }, \Delta R S$ can reach values up to $30 \%$, whereas $T F_{k}$ remains negligible.

In figure 20 we summarize the critical threshold $H_{\max }$ for all runs analysed herein. It can be seen that $H_{\max }$ is clearly more consistent in $z$ and between different runs in the intermediate region than in the inner region and the SIL. We find an average of $H_{\max }=5$ which agrees well with the value at which $T F_{k}$ begins to increase (figures 15 to 19$)$.

In figure 21 we compare total mean shear, the mean TKE flux and the net ascendant momentum associated with the LC3S for $H_{\max }$. The last quantity is represented by 

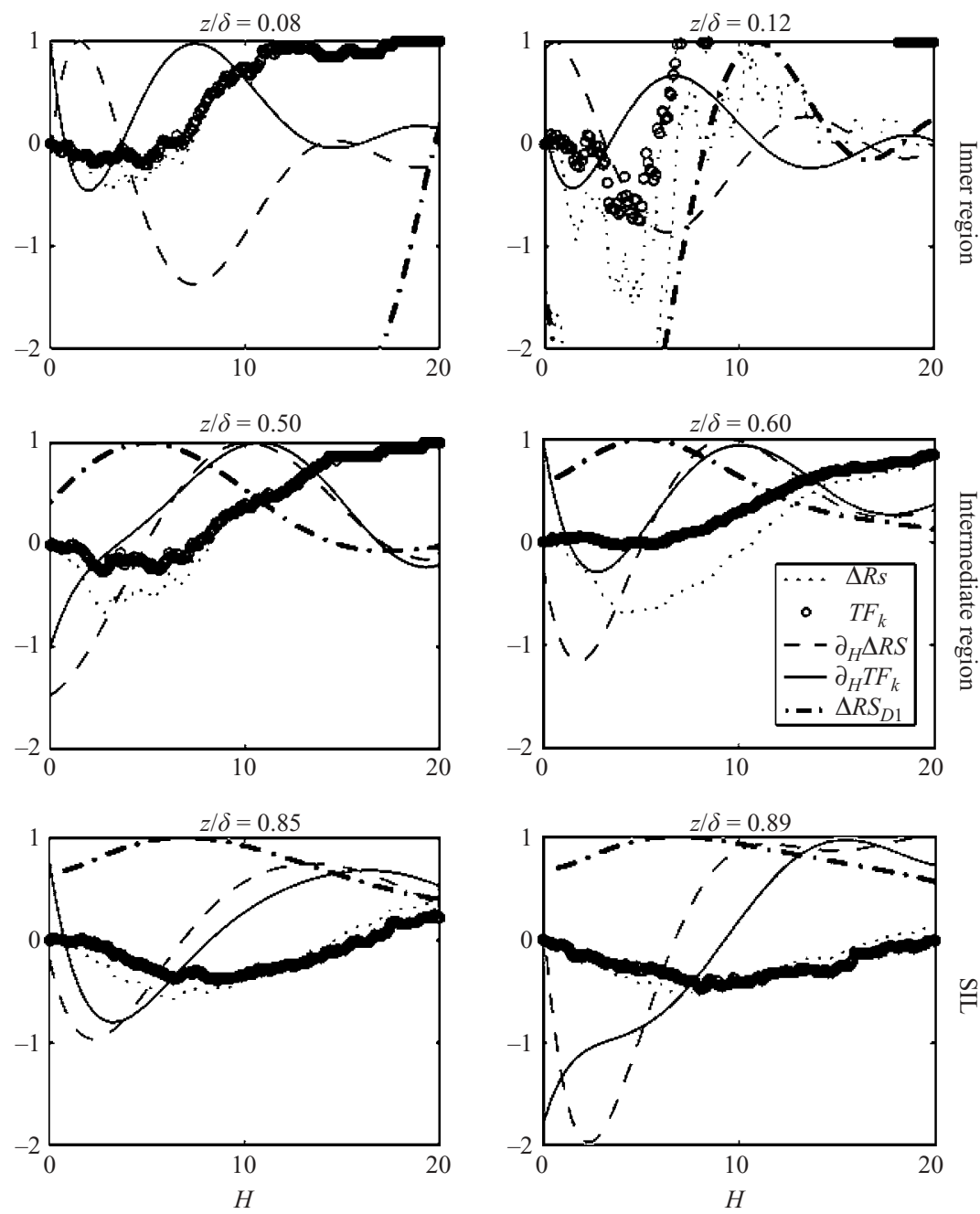

FIGURE 17. Conditionally sampled $\Delta R S, T F_{k}, \partial_{H} \Delta R S, \partial_{H} T F_{k}, \Delta R S_{D 1}$ for six different depths for run $\mathrm{C}$.

$\left(\overline{u^{\prime} w^{\prime}} / u_{*}^{2}\right) \Delta R S_{\text {max }}$. All quantities are normalized by bed friction to the power of 2 and 3 for the momentum fluxes and the mean TKE flux, respectively. For a clearer graphical presentation the mean TKE flux is plotted in the negative domain of the figure. Mean shear stress profiles are close to linear as also shown in figure 2 . The net ascendant momentum flux associated with the mean TKE flux in the intermediate region (dashed lines) represents in the mean less than $20 \%$ of the bed-friction-induced momentum flux. The spatial decay rate has the same sign as the mean shear in the SIL. It is significantly different in the intermediate region where it is close to zero for the residual momentum flux and constant with depth for the mean shear stress. The spatial decay rate of the mean TKE flux is similar to that of the residual shear stress, confirming earlier observations of a quasi-equilibrium region of the TKE flux in the intermediate region (figure $9 b$ ). Figure 20 reveals that this equilibrium originates from the nearly constant ascendant momentum flux resulting from the difference in shear associated with LC3S. In the SIL, the spatial decay rate of the mean TKE flux is 

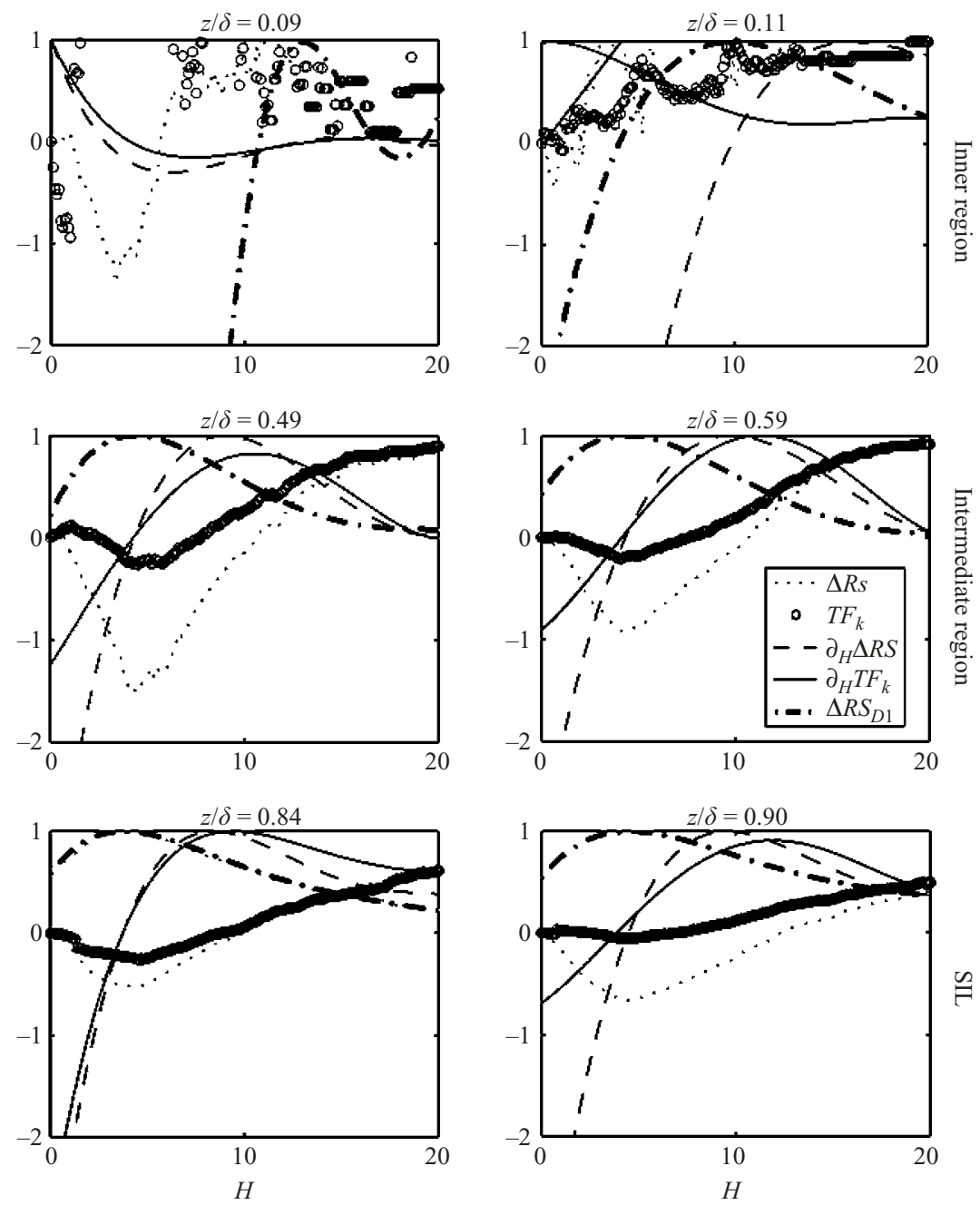

FiguRE 18. Conditionally sampled $\Delta R S, T F_{k}, \partial_{H} \Delta R S, \partial_{H} T F_{k}, \Delta R S_{D 1}$ for six different depths for run $\mathrm{AH}$.

lower for runs $\mathrm{AH}$ and $\mathrm{AL}$ than for our free-surface flows (dashed line). The absence of a free surface in free-stream boundary layers allows the LC3S to diffuse TKE over longer vertical distances than LC3S in open-channel flows.

Finally we estimate the profile of the mean vertical diffusion velocity averaged over all open-channel flow runs. This velocity takes the following form:

$$
V c=\frac{\delta}{u_{*}^{2}(\delta-z)} \frac{F_{k}}{\Delta R S_{\max }} .
$$

The solid line in figure 21 indicates the average over all runs of the diffusion velocities normalized by their bed friction velocities. We find a nearly constant diffusion velocity over the range $0.2<z / \delta<0.6$ with an average value of $1.7 u_{*}$. Below this region the velocity increases to a maximum value of $2.3 u_{*}$. Above it, it decreases with distance from the bed, reaching a value close to zero at $z / \delta=0.92$. 

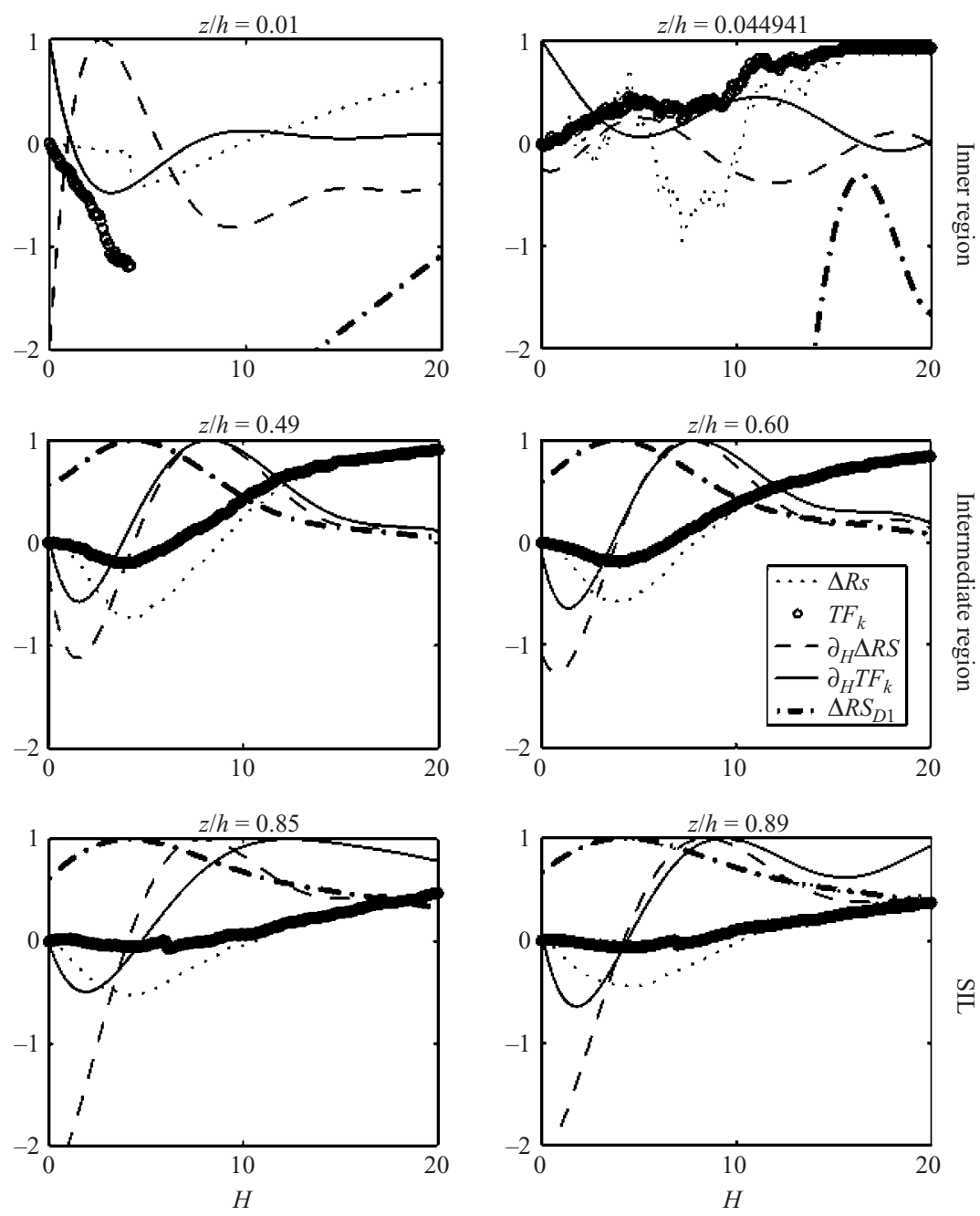

FIGURE 19. Conditionally sampled $\Delta R S, T F_{k}, \partial_{H} \Delta R S, \partial_{H} T F_{k}, \Delta R S_{D 1}$ for six different depths for run AL.

\section{Conclusion}

The present study has addressed the hydrodynamic processes related to the transport of TKE in the outer region of fully developed shear boundary layers under hydraulically rough bed conditions. The overall good concurrence in terms of dimensions and positions of large coherent shear stress structures (LC3S) relative to zones of uniform streamwise momentum strongly supports the concept of a dynamic link between LC3S and zonal organization in the outer region. LC3S can be distinguished in the instantaneous momentum flux field for quadrant threshold values of $H$ above 2. The good agreement between our quadrant analysis results and the well-documented literature results shows that LC3S identified in this study are common features in the outer region of shear flows, independent of the bed roughness.

The quantitative analysis revealed that about $20 \%$ of the shear stress contained in the LC3S generates $90 \%$ of the mean TKE flux in the outer region. This fraction of the momentum flux results from the difference between ejection- and sweep-like LC3S 


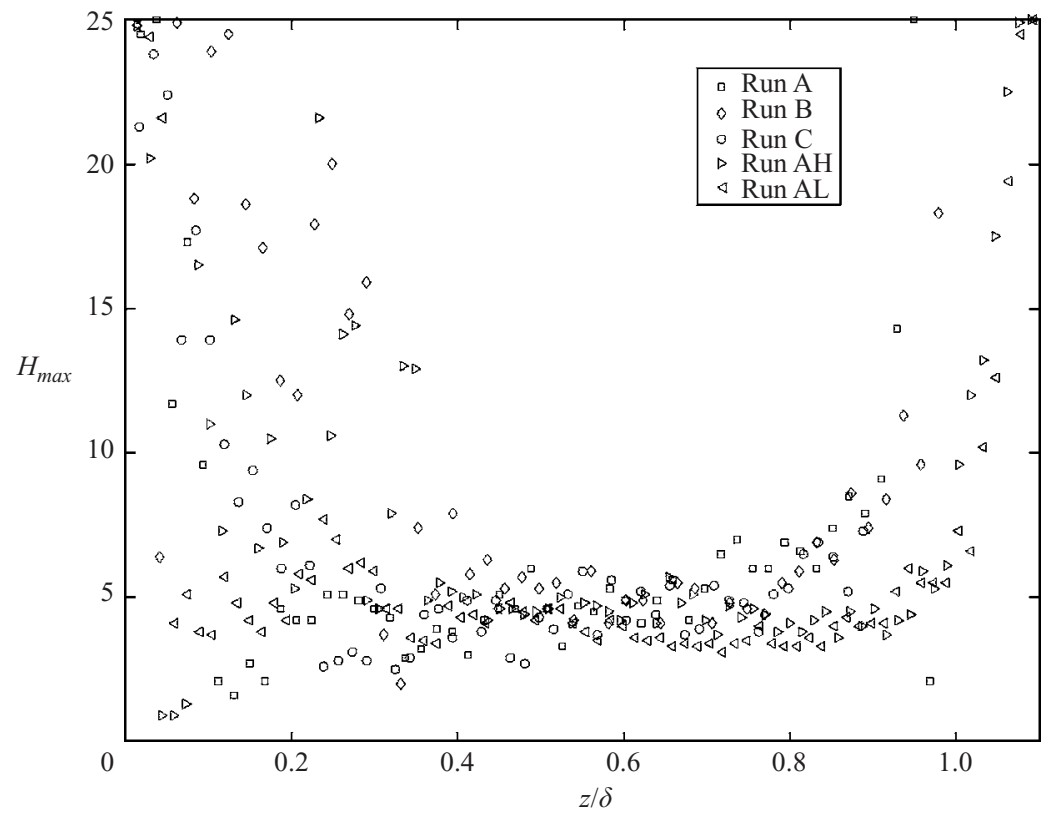

FIGURE 20. Profiles of $H_{\max }$ for all runs.

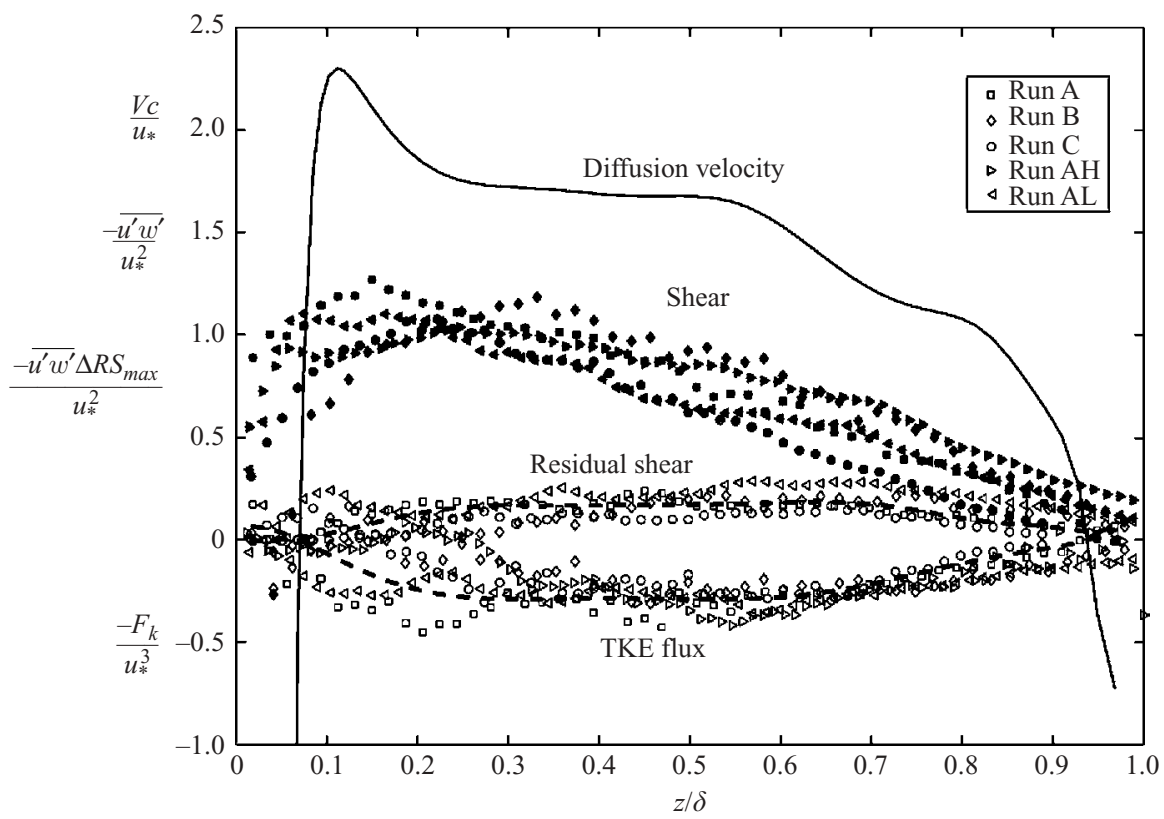

FIGURE 21. Profiles of mean shear $-\overline{u^{\prime} w^{\prime}} / u^{2}$ (in symbols with stars), residual momentum flux $\left(-\overline{u^{\prime} w^{\prime}} / u_{*}^{2}\right) \Delta R S_{\max }$ (represented by symbols in positive domain), mean vertical TKE flux $-F_{k} / u_{*}^{3}$ (represented by symbols in negative domain) and mean vertical diffusion velocity $V_{c}$ given by equation (6)

events. This residual shear stress can be estimated accurately from the local maximum of the difference between conditionally sampled ejection and sweep contributions. It is found to be ascendant over the entire outer region and nearly constant over the 
intermediate region. The mean TKE profile is also ascendant over the entire outer flow region and nearly constant over the intermediate region. The values of the critical threshold that maximize the residual shear contained in LC3S correspond to the values of the threshold that produce the mean TKE flux. The vertical profile of this critical threshold and the region of constant threshold value are consistent in the intermediate region for all flow regimes and for Adrian et al. (2000) data. In contrast, the same quantities vary significantly and randomly in the inner-flow region and in the SIL for the different runs.

The transport of TKE occurs at a quasi-equilibrium state through the intermediate region and into the SIL where it is dissipated at a nearly constant spatial decay rate. Since shear production is negligibly low in the SIL, the residual shear stress due to LC3S is the primary source of TKE. Accurate estimates of normalized TKE flux and/or the residual shear stress due to LC3S can therefore be sufficient to predict the total amount of TKE available in the SIL due to bed friction. This is an important finding with respect to those hydrodynamic processes in shallow flows which require TKE estimates in the vicinity of the free-surface, such as low-solubility gas transfer at the air-water interface, horizontal dispersion of floating material or transport of fine sediments.

We are thankful for comments of the reviewers which helped to improve the quality of the paper. The study was supported by the Swiss National Foundation for Scientific Research for the experimental part (grant 2100 050739) and the French National Center for Scientific Research (CNRS) for the data analysis and interpretation. We are grateful for their support.

\section{REFERENCES}

Adrian, R. J., Meinhart, C. D. \& Tomkins, C. D. 2000 Vortex organization in the outer region of the turbulent boundary layer. J. Fluid Mech. 422, 1-54.

Antonia, R. A. \& AtKinson, J. D. 1973 High-order moments of Reynolds shear stress fluctuations in a turbulent boundary layer. J. Fluid Mech. 58, 581-593.

BlancKaert, K. \& Lemmin, U. 2006 Means of noise reduction in acoustic turbulence measurements. J. Hydraul. Res. 44, 3-17.

Blanckaert, K. \& De VRiend, H. J. 2004 Secondary flow in sharp open-channel bends. J. Fluid Mech. 498, 353-380.

Blanckaert, K. \& DE VRIend, H. J. 2005 Turbulence structure in sharp open-channel bends. J. Fluid Mech. 356, 27-48.

Bogard, D. G. \& Tiederman, W. G. 1986 Burst detection with single-point velocity measurements. J. Fluid Mech. 162, 389-413.

Brown, G. R. \& Thomas, A. S. W. 1977 Large structure in a turbulent boundary layer. Phys. Fluids 20, 243-251.

Brumley, B. H. \& Jirka, G. H. 1987 Near-surface turbulence in a grid stirred tank, J. Fluid Mech. 183, 235-263.

Christensen, K. T. \& Adrian, R. J. 2001 Statistical evidence of hairpin vortex packets in wall turbulence. J. Fluid Mech. 431, 433-443.

Fer, I., McPhee, M. G. \& Sirevaag, A. 2004 Conditional statistics of the Reynolds stress in the under-ice boundary layer. Geophys. Res. Lett. 31, L15311, doi: 10.1029/ 2004GL020475.

Ganapathisubramani, B., Longmire, E. K. \& Marusic, I. 2003 Characteristics of vortex packets in turbulent boundary layers. J. Fluid Mech. 478, 35-46.

Garbini, J. L., Forster, F. K. \& Jorgensen, J. E. 1982 Measurement of fluid turbulence based on pulsed ultrasound techniques. Part 1. Analysis. J. Fluid Mech. 118, 445-470.

Handler, R. A., Swean Jr, T. F., Leighton, R. I. \& Swearingen, J. D. 1993 Length scales and the energy balance for turbulence near a free surface. AIAA J. 31, 1998-2007. 
Head, M. R. \& Bandyopadhyay, P. 1981 New aspects of turbulent boundary-layer structure. J. Fluid Mech. 107, 297-338.

Hurther, D. \& Lemmin, U. 1998 A constant beam width transducer for three-dimensional acoustic Doppler profile measurements in open channels. Measurement Sci. Tech. 9, 1706-1714.

Hurther, D. \& Lemmin, U. 2000 Shear stress statistics and wall similarity analysis in turbulent boundary layers using a high resolution three-dimensional ADVP. IEEE J. Ocean. Engng 25, 446-457.

Hurther, D. \& Lemmin, U. 2001 A correction method of mean turbulence measurements with a three-dimensional acoustic Doppler velocity profile. J. Atmos. Ocean. Tech. 18, 446-458.

Hurther, D. \& Lemmin, U. 2003 Turbulent particle and momentum flux statistics in suspension flow. Water Resour. Res. 39, 1139, doi:10.1029/2001WR001113.

Katul, G. G., Albertson, J., Parlange, M., Chu, C. R. \& Stricker, H. 1994 Conditional sampling, bursting, and the intermittent structure of sensible heat flux. J. Geophys. Res. D, 99, 2286922876.

Kumar, S., Gupta, R. \& BanerJee, S. 1998 An experimental investigation of the characteristics of the free surface turbulence in open channel flows. Phys. Fluids 10, 437-456.

Lhermitte, R. \& Lemmin, U. 1994 Open-channel flow and turbulence measurement by highresolution Doppler sonar. J. Atmos. Ocean. Tech. 11, 1295-1308.

López, F. \& Garcia, M. H. 1996 Turbulence structure in cobble-bed open-channel flow. Civil Engng Stud. 52, University of Illinois at Urbana-Champaign.

López, F. \& Garcia, M. H. 1999 Wall similarity in turbulent open-channel flow. J. Engng Mech. 125, 789-796.

Lu, S. S. \& Willmarth, W. W. 1973 Measurements of the structure of the Reynolds stress in a turbulent boundary layer. J. Fluid Mech. 60, 481-511.

Lui, Z., Adrian, R. J. \& Hanratty, T. J. 2001 Large-scale modes of turbulent channel flow: transport and structure. J. Fluid Mech. 448, 53-80.

MeINHART, C. D. \& AdRIAN, R. J. 1995 On the existence of uniform momentum zones in a turbulent boundary layer. Phys. Fluids 7, 694-696.

Moog, D. B. \& JIRKA, G. H. 1999 Air-water gas transfer in uniform channel flow. ASCE J. Hydraul. Engng 125, 3-10.

Nakagawa, H. \& Nezu, I. 1977 Prediction of the contribution to the Reynolds stress from bursting events in open-channel flows. J. Fluid Mech. 80, 99-128.

Nezu, I. \& NaKagawa, H. 1993 Turbulence in Open-Channel Flows. A.A. Balkema.

Nikora, V. I. \& Goring, D. G. 2000 Flow turbulence over fixed and weakly mobile gravel beds. J. Hydraul. Engng 126, 679-690.

Nimmo Smith, W. A .M., Thorpe, S. A. \& Graham, A. 1999 Surface effects of bottom-generated turbulence in a shallow tidal sea. Nature 400, 251-253.

Raupach, M. R. 1981 Conditional statistics of Reynolds stress in rough wall and smooth wall turbulent boundary layers. J. Fluid Mech. 108, 363-382.

Robinson, S. K. 1991 Coherent motion in the turbulent boundary layer. Annu. Rev. Fluid Mech. 23, 601-639.

SÉChet, P. \& Le Guennec, B. 1999 Bursting phenomenon and incipient motion of solid particles in bed-load transport. J. Hydraul. Res. 37, 683-696.

SoulsBY, R. L. 1980 Selecting record length and digitization rate for near-bed turbulence measurements. J. Phys. Oceanogr. 10, 208-219.

Theodorsen, T. 1952 Mechanism of turbulence, Proc. 2nd Midwestern Conference on Fluid Mechanics, Ohio State University, Columbus, pp. 1-18.

Thorpe, S. A., Cure, M. S., Graham, A. \& Hall, A. J. 1994 Sonar observations of Langmuir circulation and estimation of dispersion of floating particles. J. Atmos. Ocean. Tech. 11, 1273-1294.

Tomkins, C. D. \& AdRian, R. J. 2003 Spanwise structure and scale growth in turbulent boundary layers. J. Fluid Mech. 490, 37-74.

Zedel, L., Hay, A. E., Cabrera, R. \& Lohrmann, A. 1996 Performance of a single beam pulse-topulse coherent Doppler profiler. IEEE J. Ocean. Engng 21, 290-297. 